\title{
Pipelines as Communication Network Links
}

\author{
Final Report \\ for the period \\ October 1, 2002 to November 30, 2004 \\ Kelvin T. Erickson, Ann Miller, E. Keith Stanek, C. H. Wu \\ Shari Dunn-Norman \\ University of Missouri-Rolla \\ 1870 Miner Circle \\ Rolla, MO 65409 \\ U.S.A. \\ Revised \\ March 14, 2005
}




\section{DISCLAIMER}

"This report was prepared as an account of work sponsored by an agency of the United States Government. Neither the United States Government nor any agency thereof, nor any of their employees, makes any warranty, express or implied, or assumes any legal liability or responsibility for the accuracy, completeness, or usefulness of any information, apparatus, product, or process disclosed, or represents that its use would not infringe privately owned rights. Reference herein to any specific commercial product, process, or service by trade name, trademark, manufacturer, or otherwise does not necessarily constitute or imply its endorsement, recommendation, or favoring by the United States Government or any agency thereof. The views and opinions of authors expressed herein do not necessarily state or reflect those of the United States Government or any agency thereof." 


\begin{abstract}
This report presents the results of an investigation into two methods of using the natural gas pipeline as a communication medium. The work addressed the need to develop secure system monitoring and control techniques between the field and control centers and to robotic devices in the pipeline. In the first method, the pipeline was treated as a microwave waveguide. In the second method, the pipe was treated as a leaky feeder or a multi-ground neutral and the signal was directly injected onto the metal pipe. These methods were tested on existing pipeline loops at UMR and Batelle. The results reported in this report indicate the feasibility of both methods. In addition, a few suitable communication link protocols for this network were analyzed.
\end{abstract}




\section{TABLE OF CONTENTS}

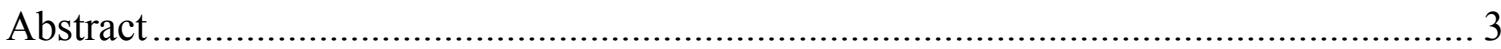

TABLE OF CONTENTS

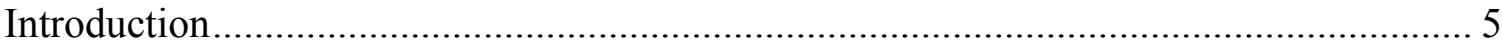

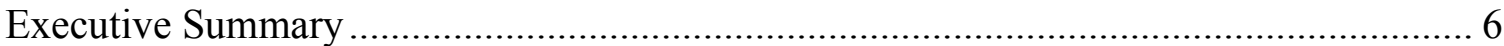

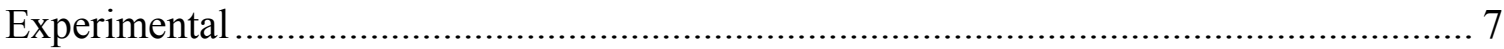

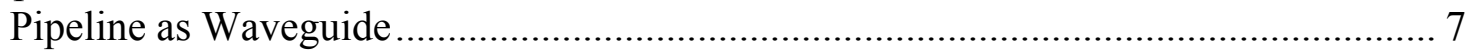

Verify attenuation of electromagnetic waves in a pipeline..................................... 7

Measure transmission loss of pipeline.................................................................. 13

Use commercial wireless modems on test pipelines............................................. 14

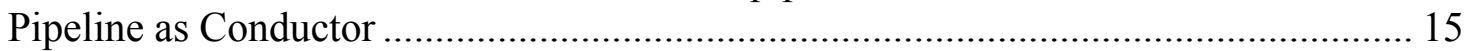

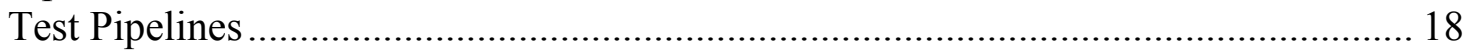

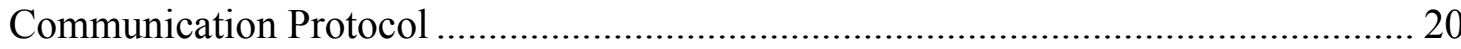

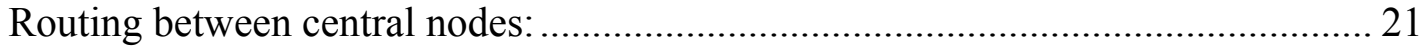

Routing between nodes within a subnet: ............................................................. 21

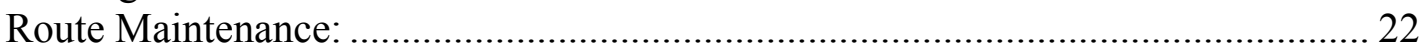

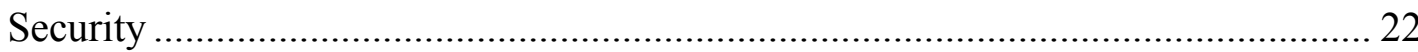

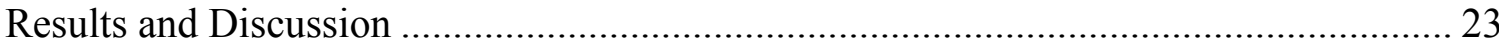

Pipeline as Waveguide ......................................................................................... 23

Verify attenuation of electromagnetic waves in a pipeline....................................... 23

Measure transmission loss of pipeline................................................................. 26

Use commercial wireless modems on test pipelines............................................... 28

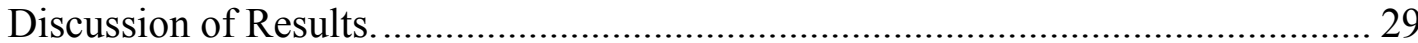

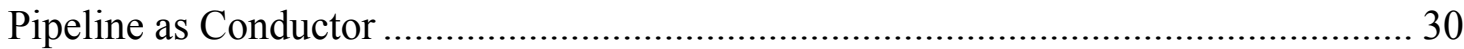

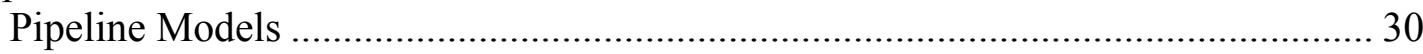

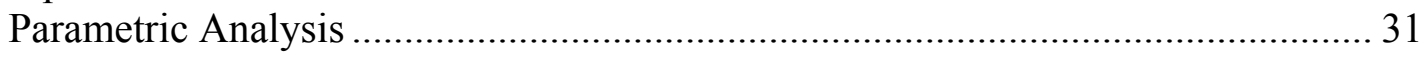

Attenuation Measurements ................................................................................. 39

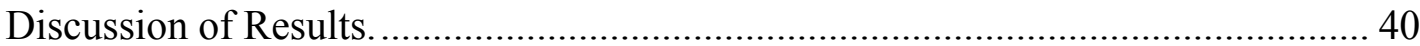

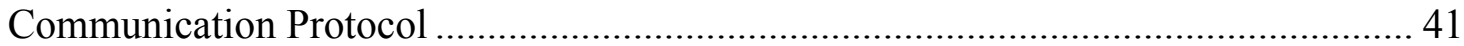

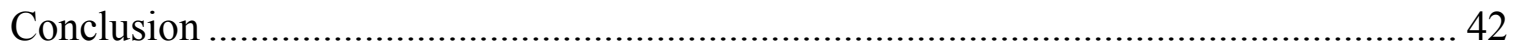

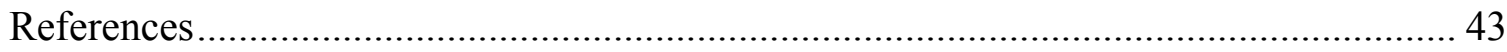

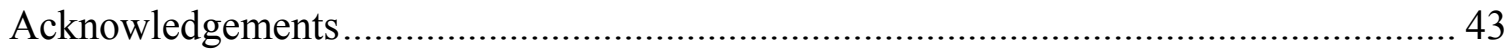

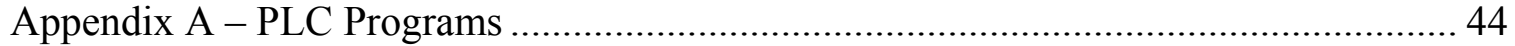

Appendix B - Parametric Study Program.................................................................. 48 


\section{Introduction}

The existing U.S. natural gas pipeline transmission network is comprised of approximately 90 pipeline systems that make up the onshore mainline interstate gas pipeline network. Another $60+$ pipeline systems make up the gathering lines. The majority of these natural gas pipeline systems are large diameter (>30") steel pipelines with welded joints. Gathering lines are similar, although these lines typically are smaller in diameter.

Gas is routed from a field source or gas processing plant to the end user through a system of pipelines.

Gas movement through these pipeline systems is accomplished by adding compression stations approximately every 50 to 100 miles. In addition, safety shut-in valves may be included at critical places in a pipeline, particularly near high consequence areas. Pipeline pressures and flows are typically monitored and/or controlled with SCADA systems using remote terminal units (RTU), programmable logic controllers (PLCs), and either satellite, microwave, or telephone communication links. These controls are subject to interruption from loss of communication and are subject to interference by knowledgeable third party intruders.

Reliable communications links from one end of a pipeline to the other end are vital for effective pipeline monitoring and control. In addition, reliable communications is needed to support robotic devices residing in the pipeline that diagnose and repair pipeline problems. This report presents the preliminary results of two methods of using the natural gas pipeline as a communication medium:

- Pipe as waveguide for microwaves or commercial wireless modems

- Pipe as signal conductor.

All of these methods may be retrofitted into existing pipeline infrastructure.

Regardless of which technology is selected for the implementation of the communication links, the end result will be sets of intra-pipeline communication "chains" which are connected to a monitoring and control "hub". A few suitable communication link protocols for this network are analyzed. In addition to analyzing the communication link technology, the stability, security, and survivability of the entire communication system is also considered. 


\section{Executive Summary}

This work addressed the need to develop secure system monitoring and control techniques between the field and control centers and to robotic devices in the pipeline. Reliable communications links from one end of a pipeline to the other end are vital for effective pipeline monitoring and control. In addition, reliable communications is needed to support robotic devices residing in the pipeline that diagnose and repair pipeline problems. This report presents the preliminary results of two methods of using the natural gas pipeline as a communication medium:

- Pipe as waveguide for microwaves or commercial wireless modems

- Pipe as signal conductor.

Both of these methods may be retrofitted into existing pipeline infrastructure.

Regardless of which technology is selected for the implementation of the communication links, the end result will be sets of intra-pipeline communication "chains" which are connected to a monitoring and control "hub". A few suitable communication link protocols for this network are analyzed. In addition to analyzing the communication link technology, the stability, security, and survivability of the entire communication system is also considered.

For waveguide transmission, the propagation characteristics of the pipeline were experimentally determined by evaluating the attenuation of a 3.5 meter section of pipe. For the pipe as a signal conductor, the pipeline was modeled as a distributed electrical circuit whose attenuation characteristics were evaluated for ranges of the parameters. Both of these initial evaluations showed the expected range of transmission frequencies for which the medium can effectively transmit over long distances.

Both transmission methods were initially verified on a small pipeline loop at the University of Missouri-Rolla. The pipeline as a waveguide effectively transmitted signals of a few $\mathrm{GHz}$. A test with commercial $802.11 \mathrm{~b}$ modems was also successful. When using the pipeline as a conductor, the UMR pipeline showed little attenuation.

Both methods were also tested with a much longer pipeline at Battelle. The pipeline as a waveguide effectively transmitted signals of around $1 \mathrm{GHz}$, a lower frequency than for the UMR pipeline. Tests with commercial $802.11 \mathrm{~b}$ modems were also successful. When using the pipeline as a conductor, the Battelle pipeline could transmit signals in the range of 10 to $100 \mathrm{kHz}$.

From the communication protocol simulations, Optimized Link State Routing (OLSR) performed the best for a large scale network of pipeline sensors. While the data delivery rate was not quite as high as one would hope, especially at higher node speeds, delivery rate probably could be increased with a change of the OLSR parameters to allow more frequent neighbor sensing and multipoint relay set broadcast. With small networks, Dynamic Source Routing (DSR) would probably be a better choice because of its high data delivery rate.

The experimental results presented in this report indicate that both methods of transmitting signals are feasible. A pipeline can act as a waveguide to frequencies in the few $\mathrm{GHz}$ range, allowing the use of commercially-available radio modems. A pipeline can also support direct signal injection of a signal with a frequency of a few $\mathrm{kHz}$. The next step in this work is to build hardware to test the feasibility to transmit a few miles. 


\section{Experimental}

The theoretical analysis and experimental methods are described for testing the pipeline as a waveguide and as a conductor. In addition, the test pipelines are described. Completing this section is a description of the communication protocols that will support a network of sensors.

\section{Pipeline as Waveguide}

An example installation is shown in Figure 1. Radio signals are used to transmit information between nodes. The antenna for each node is shown inside a non-metallic shield called a radome. The radome allows replacement of the antenna without opening the pipeline. To assess the feasibility of wireless communication within a natural gas pipeline, the overall experimental procedure was as follows:

1. Experimentally verify the attenuation of electromagnetic waves in a pipeline.

2. Experimentally measure the transmission loss of a 6" pipeline at UMR and a 24 " pipeline at Battelle.

3. Use commercial wireless modems to verify that the test pipelines can support wireless communication with commercial-off-the-shelf (COTS) equipment.

Each of these overall steps is explained below.

\section{Verify attenuation of electromagnetic waves in a pipeline.}

Radio wave transmission in free space is well known. In free space, radio waves are in the form of a transverse electromagnetic wave (TEM wave), which means that propagation direction, electric field direction, and magnetic field direction are perpendicular to each other. The nature of a wave implies that both electric and magnetic field amplitudes are oscillating with time at a given frequency. Thus, as a TEM wave is propagating in a given direction in a medium, such as air, having a small conductivity, the oscillating electric field generates an oscillating current in the same direction. This generation is due to Ohm's law. The net result of

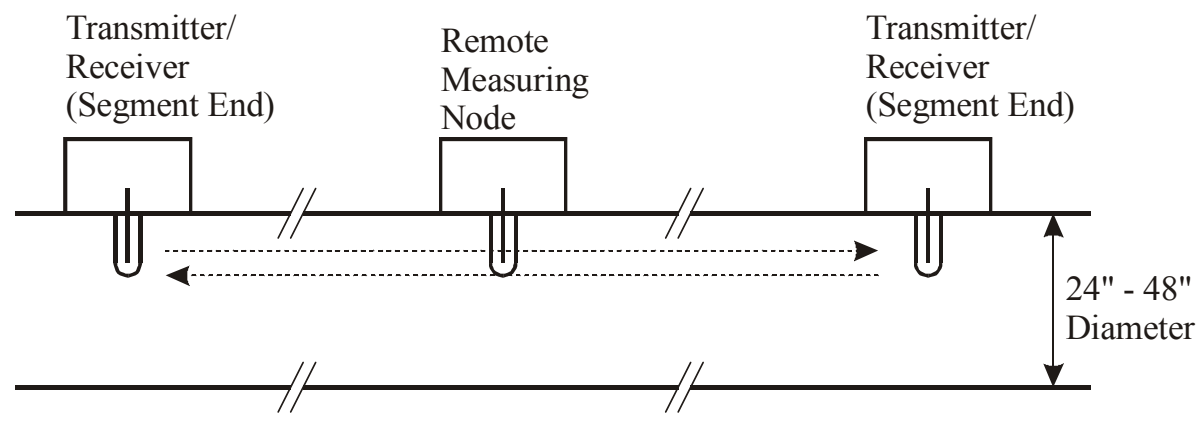

Figure 1. Pipeline as waveguide. 
this generation causes the electric field (or the magnetic field) to be weakened (or attenuated) as the wave is propagating along. The attenuation of a TEM wave can be easily measured. Thus, for a given antenna that beams a TEM wave in a favored direction through the air, the transmission distance can be established through the transmitting power, the attenuation constant through the air (in the units of $\mathrm{dB}$ per meter or kilometer), and the minimum power requirement for the receiving antenna system at a given frequency. Now consider the propagation of an electromagnetic wave in a straight gas pipeline. A pipeline is a waveguide. There can be no TEM wave in any waveguides, because inside the pipe, a TEM wave has a transverse variation like a static field and no static fields can exist inside the region bounded by the pipe. However, transverse electric waves (TE mode waves) or transverse magnetic waves (TM mode waves) can exist in a pipeline. In a TE wave, only the electric field is perpendicular to the propagation direction while the magnetic field can now be in the propagation direction. Furthermore, the frequency that can support propagation in a pipeline has to be higher than a certain frequency, called the cut-off frequency, unlike TEM waves. So usually, there is a lowest allowed frequency that can propagate in a pipe and then the next highest frequency and so on. They form a discrete set of frequencies, which is labeled as $T E_{m n}$ or $T M_{m n}$ modes, where the indices, $m n$, are integers to indicate the mode. A straight pipeline is a circular waveguide and oversized. The allowed frequencies have been calculated for a given diameter and material of the pipe. Those results can be found in a typical textbook (for example, Balanis, 1989). The $T E_{11}$ mode has the lowest cutoff frequency and the $T E_{01}$ mode has the electric field lines not terminating on the wall of a pipeline and hence this mode suffers very little loss due to the conducting wall of the pipe. Therefore, the $T E_{01}$ mode is an ideal mode to launch in a pipeline because of the low attenuation.

The above description points out the fact that when a radio wave propagates inside a pipeline, the main loss is due to the thin penetration and hence the disappearance of the wave into the steel pipe. The air or the gas plays a minor role, which is different from the situation of propagation in free space. The question is then how to first evaluate the loss when the pipe is straight. Additional bends will be considered after that.

The methods of our investigations are divided into two parts:

(1) Transmission line method: As described earlier, a pipeline will not support a TEM mode transmission and hence the typical method of evaluating transmission loss in free space can not be used directly. However, if one inserts a copper tube in the center of the pipeline, then the two concentric pipes form a "giant coaxial cable". The wave propagation in a coaxial cable will support a TEM mode and the attenuation constant is well known and can be measured. However, the transmission loss in this case will be due to both the copper tube and the steel pipeline. But, because the transmission loss due to copper material is three orders of magnitude less than that of steel from the values of their conductivity, one can safely assume that the major loss is from the steel wall. Ideally, one should use a very long pipeline with an inserted copper pipe to form a very long coaxial cable. With the TEM wave generated at one end, one can measure the attenuation at the other end just like a typical TEM wave loss measurement in a free space. But such a long and giant coaxial cable is not practical to make. So an equivalent method was devised. We used a cone shaped section that is made out of copper to launch a TEM wave at one end. By the time the wave arrives at the beginning part of "a short coaxial cable", a TEM wave is there and starts to 
propagate through the short section of the cable. When the wave arrives at the other end of the cable, another copper cone converges the wave into a point and measures the attenuation loss. So the two copper cones used to diverge and converge a plane wave is similar to the use of two lenses spaced at a distance to move monochromatic light. The source and measurement are located at the focal point of each lens. We have assumed that the loss due to the copper cone sections is at a minimum and the measured loss is due to the transmission loss from the steel pipe.

(2) Resonance cavity method: If one tightly seals the two ends of a pipeline with two copper plates, then the whole space inside the steel pipe becomes a resonant cavity. If one generates an electromagnetic wave inside the cavity (by inserting a dipole or a probe, for example) and store the energy inside the box and then watch how many times (the quality factor, or the $\mathrm{Q}$ factor of a resonator) the wave can bounce inside the cavity before the wave dies out, we can then relate the transmission loss due to the steel pipe as well. After all, each time the wave bounces the steel wall assuming the two copper end plates incur no loss at all), a loss is occurred and after certain amount of bounces, all the wave energy is lost to the steel wall of the pipeline. But remember that a resonant cavity supports a discrete set of "resonant frequencies" only because now the wave is stationary and not propagating. The frequency is labeled as $f_{m n l}$ where the indices $m n l$ are from either the $T E_{m n l}$ mode or from the $T M_{m n l}$ mode. The derivation of the resonant frequencies and the $\mathrm{Q}$ factor for a circular cylindrical resonator is available. The first two indices $m n$ are the mode indices (two integers) for the cross section of the pipe and the last index $l$ is the mode index (or an integer) for the length of the pipe in a given resonant mode. The $\mathrm{Q}$ factor measured is then related to the transmission loss.

Both of these methods are described next.

\section{Transmission Line Method}

The pipe is used as the outer conductor of the TEM structure and an inner conductor need to be added. To give a characteristic impedance of 50-ohmn matching with the measurement system, the ratio of the radius of the outside conductor to the inner conductor should be around 2.6. As illustrated in Figure 2, the inner conductor is a bundle of copper cubes. At the two ends of the pipe, core shape structures are used to bring the pipe size down to the SMA connectors and try to maintain a characteristic impedance at about 50-ohm (Figure 3). Figure 4 shows the measurement setup for the insertion loss through the pipe. $20 \mathrm{~dB}$ attenuators are added on both ends of the pipe to match the measurement system. A vector network analyzer is the measuring instrument. The procedure is as follows:

1. Use the time-domain reflectometer mode of the network analyzer to measure the characteristic impedance of the resulting structure to verify that it is close to 50 ohms.

2. Use the network analyzer to measure the return loss, which is the ratio of the magnitude of the reflected power divided by the magnitude of the incident power, for a range of frequencies. 


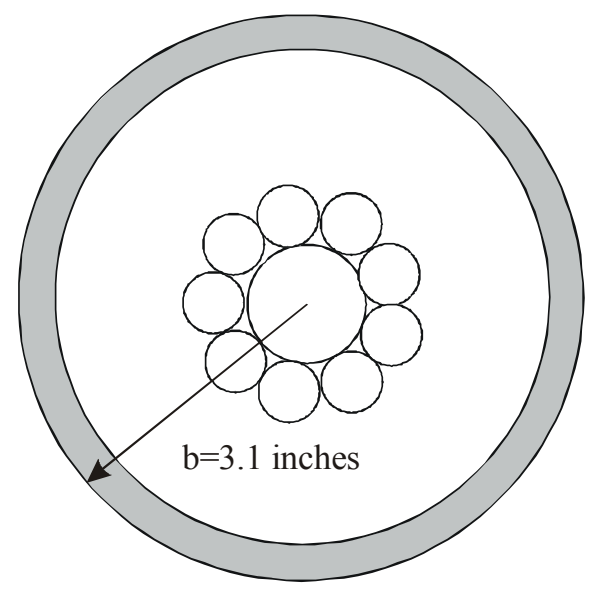

Figure 2. Transection view of the structure for TEM mode.

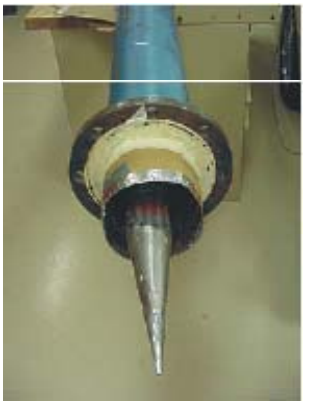

(a)

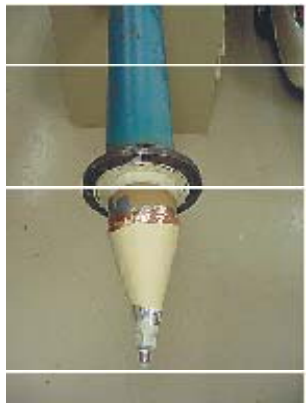

(b)

Figure 3. Core shape transition from pipe to SMA connector: (a) inside; (b) outside. 


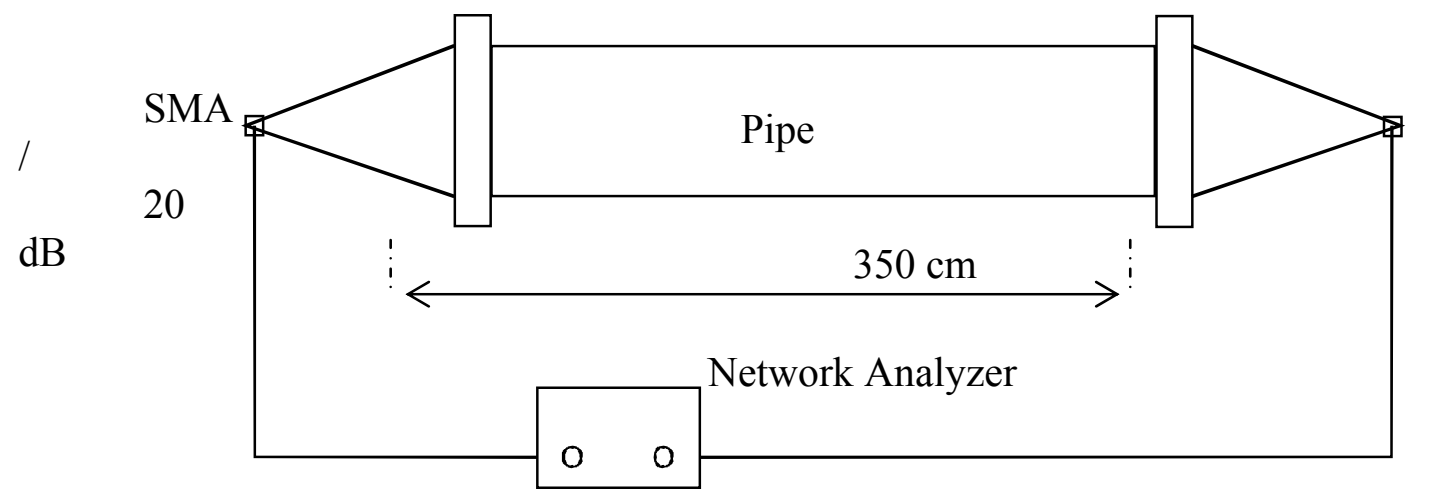

SMA/

$20 \mathrm{~dB}$

Attenuator

Figure 4. Transmission line measurement setup

\section{Resonance Cavity Method}

To find the resonant frequencies and the $\mathrm{Q}$ factor for the evaluation of the transmission loss in a pipeline, one must first set up a structure supporting a transverse electromagnetic (TEM) waveguide, as shown in Figure 5. The pipe was electrically shorted at both ends to make it a cylindrical cavity resonator. Copper taps make very good electrical contact considering the operating frequency of the wave in the test.

Since the dominant mode in a circular waveguide is the $\mathrm{TE}_{11}$ mode ( with a lowest cut-off frequency), the dominant cylindrical cavity resonant mode is the $\mathrm{TE}_{111}$ mode. The $\mathrm{TE}_{111}$ can be excited by a probe connected to the input through a coaxial cable (labeled "SMA" in Figure 5). With the probe set in the middle of the pipe in the longitudinal direction, only the odd resonant modes of $\mathrm{TE}_{11 l}$ can exist.

The resonant frequency of the $\mathrm{TE}_{m n l}$ mode can be calculated as

$$
f_{m n l}=\frac{c}{2 \pi \sqrt{\mu \varepsilon}} \sqrt{\left(\frac{p_{m n}}{a}\right)^{2}+\left(\frac{l \pi}{d}\right)^{2}}
$$

where $c$ is the speed of light, $\mu$ is the magnetic permeability of the air, $\varepsilon$ is the dielectric constant of the air, $p_{m n}$ is the constant associated with the boundary condition of the circular cross section of radius $a$, (for example, $p_{01}=2.405$ ), $a$ is the pipe inner radius, and $d$ is the length of the cavity.

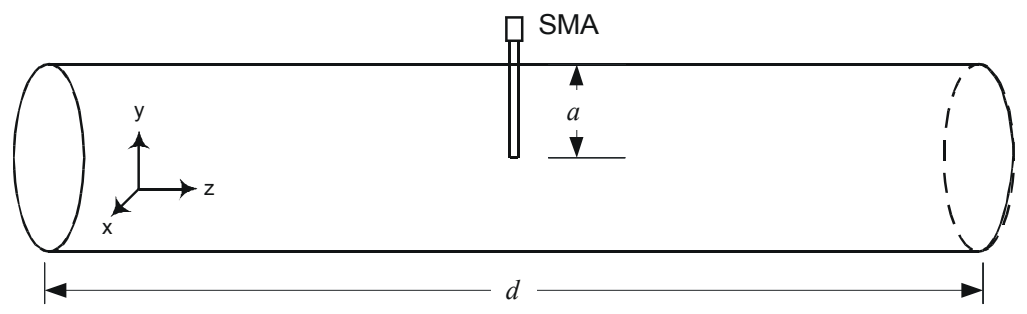

Figure 5. The gas pipeline as a cylindrical resonant cavity (size in meters) 
The resonant frequency is the frequency at which the best transmission occurs. However, to determine how effective the pipeline is as a waveguide, the conducting loss coefficient must be measured. The conducting loss is determined in the following manner, called the Q-factor test. After explaining the theoretical basis for the calculations, the procedure is summarized.

The return loss, $\mathrm{S}_{11}$, which is the ratio of the magnitude of the reflected power divided by the magnitude of the incident power, must be measured for a range of frequencies around the resonant frequency. The return loss shows the percentage of reflecting power versus incident power. So, from the return loss measurement,

$$
P_{\text {refl }}=\left.\Gamma\right|^{2} P_{i n c}
$$

where $|\Gamma|^{2}$ is the return loss. Then the power absorbed in the resonator at a particular frequency is

$$
\frac{P(f)}{P_{i n c}}=\frac{P_{i n c}-P_{r e f l}}{P_{i n c}}=1-|\Gamma|^{2}
$$

where $P_{i n c}$ is the incident power and $P_{\text {refl }}$ is the reflected power. Given that the power absorbed at the resonant frequency is $P\left(f_{0}\right)$, then at the frequencies for which the return loss is $3 \mathrm{~dB}$ less (half of the power absorbed) points, we have:

$$
\frac{P\left(f_{3 d B}\right)}{P\left(f_{0}\right)}=\frac{1-\left|\Gamma\left(f_{3 d B}\right)\right|^{2}}{1-\left|\Gamma\left(f_{0}\right)\right|^{2}}=\frac{1}{2}
$$

The $3 \mathrm{~dB}$ frequencies are on either side of the resonance frequency. The difference between these two frequencies is $\Delta f$. Then the load $\mathrm{Q}$-factor $\mathrm{Q}_{\mathrm{L}}$, and no-load $\mathrm{Q}$-factor $\mathrm{Q}_{0}$, is given as:

$$
\begin{aligned}
& Q_{L}=\frac{f_{0}}{\Delta f} \\
& K=\frac{1+\left|\Gamma\left(f_{0}\right)\right|}{1-\left|\Gamma\left(f_{0}\right)\right|} \\
& Q_{0}=(K+1) Q_{L}
\end{aligned}
$$

where, $f_{0}$ is the resonant frequency and $\Delta f$ is the $3 \mathrm{~dB}$ bandwidth.

The conducting loss coefficient of the pipe can be calculated as:

$$
\begin{aligned}
& Q=\frac{(k a)^{3} \eta a d}{4\left(P_{m n}^{\prime}\right)^{2} R_{s}} \frac{1-\left(\frac{m}{\left(P_{m n}^{\prime}\right)^{2}}\right.}{\left\{\frac{a d}{2}\left[1+\left(\frac{\beta a m}{\left(P_{m n}^{\prime}\right)^{2}}\right)^{2}\right]+\frac{\left(\beta a^{2}\right)^{2}}{\left(P_{m n}^{\prime}\right)^{2}}\left(1-\left(\frac{m}{\left(P_{m n}^{\prime}\right)^{2}}\right)\right\}\right.} \\
& R_{s}=\sqrt{\frac{\omega \mu}{\sigma_{c}}}
\end{aligned}
$$

where

$$
k=\omega \sqrt{\mu \varepsilon} \quad \text { and } \quad \beta=\sqrt{k^{2}-\frac{\left(P_{m n}^{\prime}\right)^{2}}{a^{2}}} .
$$

Thus, the loss coefficient $\Gamma\left(f_{0}\right)$ at resonant frequency $f_{0}$ can be evaluated from the above.

Summarized, the procedure is as follows 
1. Measure the coupling factor of the resonator by observing the resonant peak value.

2. Use a vector network analyzer to measure the return loss, $\mathrm{S}_{11}$, which is the ratio of the magnitude of the reflected power divided by the magnitude of the incident power, for a range of frequencies around the resonant frequency.

3. Calculate and plot the return loss data as $\left(1-\left|\Gamma\left(f_{0}\right)^{2}\right|\right)$

4. Find the $3 \mathrm{~dB}$ bandwidth on the $\left(1-\left|\Gamma\left(f_{0}\right)^{2}\right|\right)$ plot.

5. Calculate the $\mathrm{Q}_{\mathrm{L}}$ and $\mathrm{Q}_{0}$.

6. Calculate the conducting loss coefficient.

After measuring the attenuation loss in $\mathrm{dB} / \mathrm{m}$ for a straight pipeline through the above two methods, one needs to assess the additional losses due to the bends and other imperfections that exist in a real pipeline. Those losses are difficult to evaluate analytically. The next major step accomplished this task.

\section{Measure transmission loss of pipeline.}

The transmission loss of two pipelines (one at UMR and the other at Battelle) was measured. The pipelines are described below. The procedure to measure the transmission loss is described here.

To measure the transmission loss, one uses a vector network analyzer (VNA). In this case, the Hewlett-Packard 8753D vector network analyzer was used. To use the VNA to measure the transmission loss, one sets up two antennas, at either end of the pipeline (Figure 6), one connected to Port 1 of the VNA and the other connected to Port 2 of the VNA. The antenna at Port 1 transmits the signal and the antenna at Port 2 receives the signal. The received signal strength (volts/meter) divided by the transmitted signal strength (volts/meter) is the gain of the transmission system (pipeline, in this case).

$$
\text { Gain }=(\text { Received signal strength }) /(\text { Transmitted signal strength })=v_{d} / v_{i}
$$

The gain is normally reported in $\mathrm{dB}$,

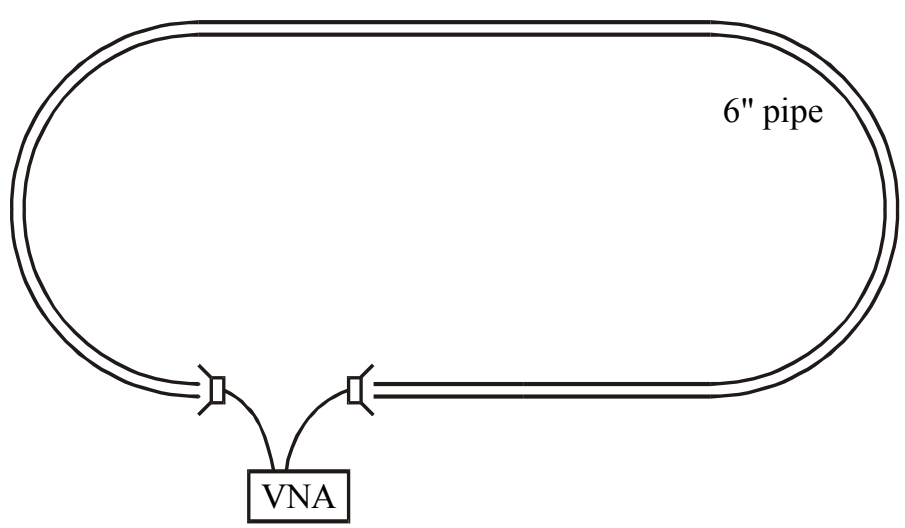

Figure 6. Waveguide test setup. 


$$
\text { Gain }(\mathrm{dB})=20 \log _{10}\left(v_{o} / v_{i}\right)
$$

The receiver antenna was a double-ridged horn antenna and the transmitter antenna was a dish/feed antenna. Both antennas had almost uniform gain and nominal $50 \mathrm{ohms}$ impedance over the range of operating frequencies. Absorbing material is used in the air gap between the antennas to isolate the two antennas and to minimize the crosstalk between the two antennas.

The noise floor is determined when the two antennas are pointing away from each other in open air. This test measures the background electromagnetic signals.

\section{Use commercial wireless modems on test pipelines.}

After initial tests showed that the pipeline showed good transmission characteristics up to a few $\mathrm{GHz}$, commercial $802.11 \mathrm{~b}$ modems were also tested with the pipelines. This particular test was either a go/no-go type of test. No attempt was made analyze the signals in the pipeline.

Locus, Inc. model 2400-HSE modems were connected to Ethernet programmable logic controllers (PLC) that were sending short messages to each other. The PLC programs are shown in Appendix A. When communication is successful, the O:2/00 output indications on each PLC flash at a $0.5 \mathrm{~Hz}$ rate. The particular modems transmit a 0.25 watt signal. The test setup is shown in Figure 7. Both antennas were placed inside the pipe. One modem was placed inside the pipe and the other modem was left outside the pipe so that the communication status can be monitored. As for the transmission loss test, the pipeline ends were completely sealed with aluminum foil and electromagnetic absorbing foam.

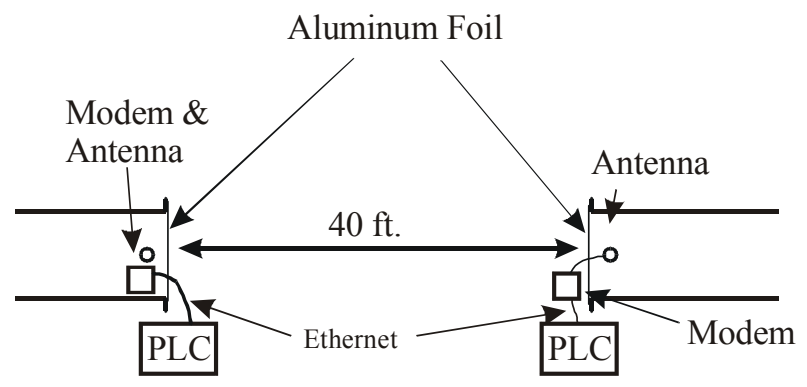

Figure 7. Test setup with wireless modems. 


\section{Pipeline as Conductor}

At first glance, using the pipe as a conductor (Figure 8) seems an unlikely candidate for a communications channel since the pipeline is frequently buried or at least laying on the earth. However, these pipelines are generally epoxy coated to retard corrosion. Therefore, the contact to earth is minimal over the bulk of the pipe's surface. It is recognized that there will be intermittent contact of the pipeline with the earth, especially with cathodic protection. This situation closely matches the neutral of a transmission or distribution line that is intermittently grounded at each pole or at alternate poles. These neutral conductors are still used to transmit signals in some relaying and metering schemes. This transmission is possible because the connections to earth are of relatively high resistance due to a combination of high earth resistivity and a small amount of metallic contact area with the earth.

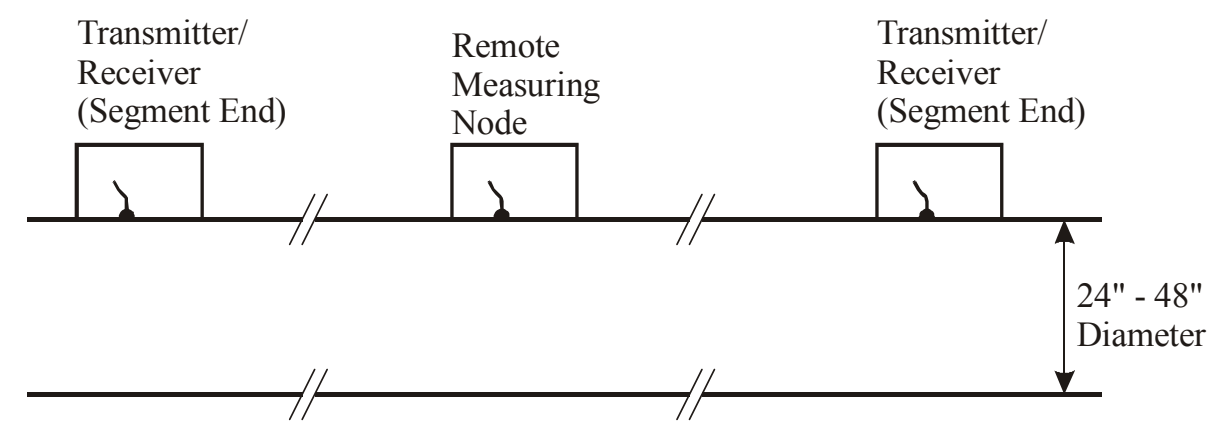

Figure 8. Pipeline as conductor, direct signal injection

A transmission system with a distributed ground can be modeled as shown in Figure 9. In this figure, $R$ is the series resistance per unit length of the conductor (the pipeline) in $\Omega / \mathrm{mile}, L$ is the inductance per unit length of the conductor (pipeline) in $H /$ mile, $C$ is the capacitance per unit length of the conductor (pipeline) in $F /$ mile, and $G$ is the conductance per unit length of the conductor (pipeline) in siemens/mile.

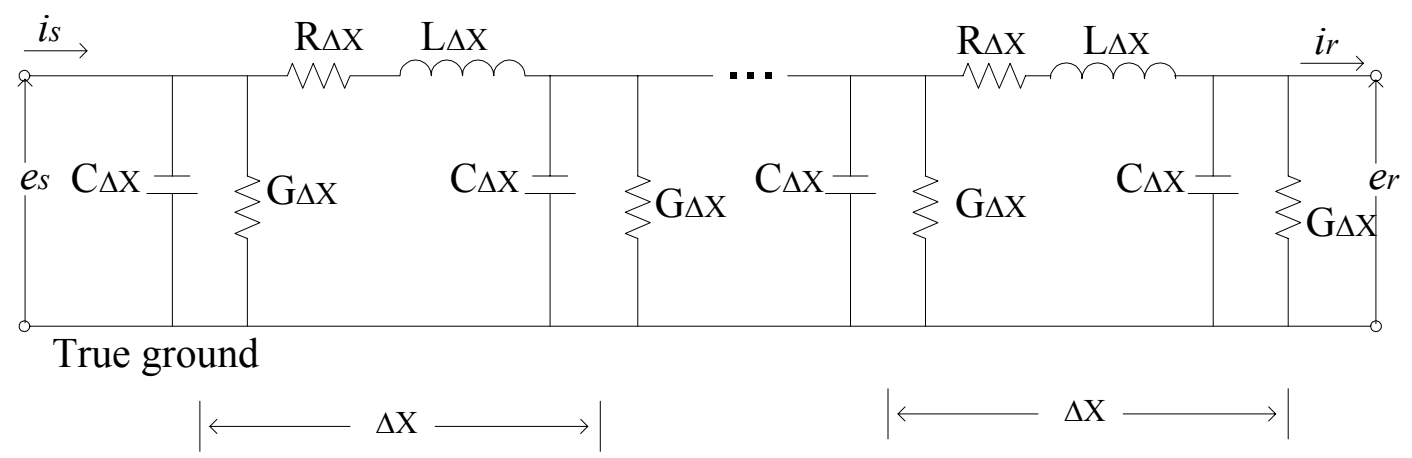

Figure 9. Electrical Model of a Distributed, Continuously Grounded Pipeline. 
The electrical performance of this line is basically that of a lossy transmission line. The performance can be summarized by the following equations obtained by writing Kirchoff's voltage and current laws for a section of line of length $\Delta \mathrm{x}$ and taking the limits as $\Delta \mathrm{x} \rightarrow 0$

$$
\begin{aligned}
& \frac{\partial e}{\partial x}=-R i-L \frac{\partial i}{\partial t} \\
& \frac{\partial i}{\partial x}=-G e-C \frac{\partial e}{\partial t}
\end{aligned}
$$

In the sinusoidal steady-state analysis

$$
\begin{gathered}
e(x, t)=E(x) e^{j \omega t}=E e^{j \omega t} \\
i(x, t)=I(x) e^{j \omega t}=I e^{j \omega t}
\end{gathered}
$$

Thus,

$$
\begin{aligned}
& \frac{d E}{d x} e^{j \omega t}=-(j \omega L+R) I e^{j \omega t} \\
& \frac{d I}{d x} e^{j \omega t}=-(j \omega C+G) E e^{j \omega t}
\end{aligned}
$$

If equation (1) is differentiated with respect to $\mathrm{x}$ and combined with equation (2) the following is obtained

$$
\frac{d^{2} E}{d x^{2}} e^{j \omega t}=(R+j \omega L)(G+j \omega c) E e^{j \omega t}
$$

Eliminating the common factor of $e^{j \omega t}$ yields

$$
\frac{d^{2} E}{d x^{2}}=(R+j \omega L)(G+j \omega c) E
$$

The solution to this equation is

$$
E(x)=E_{o}^{+} e^{-\gamma x}+E_{0}^{-} e^{+\gamma x}
$$

where

$$
\gamma=\sqrt{(R+j \omega L)(G+j \omega C)}
$$

Re-introducing the factor of $e^{j \omega t}$ yields

$$
e(x, t)=E_{o}^{+} e^{j \omega t-\gamma x}+E_{o}^{-} e^{j \omega t+\gamma x}
$$

In equation (3), the first term on the left hand side is a forward traveling wave and the second term on the left hand side is a reverse traveling wave. 
The propagation of signals on this lossy line depends strongly on the values of $R$ and $G$. If both are zero, then $\gamma$ is imaginary and $\gamma x$ represents a phase shift but there is no attenuation. When either $R$ or $G$ is non-zero, $\gamma$ has a positive real part and $e^{-\gamma x}$ represents both a phase shift and attenuation. Clearly, finding the effective parameters of the pipeline $(R, G, L, C)$ is a key step in the assessment of how well signals can be propagated. It should also be clear that $\gamma$ is highly frequency dependent, so communications between ends of the pipeline will be more feasible at some frequencies than others.

There are a significant number of physical characteristics that affect the line parameters $(\mathrm{R}, \mathrm{L}, \mathrm{C}, \mathrm{G})$ of a pipeline. These include pipe diameter, wall thickness, height of the pipe's center with respect to the earth surface, frequency, earth resistivity, pipeline material, and coatings on the pipeline's surface. Of these, the most important are earth resistivity, frequency and pipeline material. Other parameters have little effect on the fine parameters and hence the attenuation per unit length. One of the reasons for this is that some of the dimensional characteristics only occur in the arguments of log functions.

Initially, typical values of earth resistivity, pipeline material (cast iron and stainless steel) and coatings were used to calculate the expected attenuation of the pipeline at frequencies up to 1 MHz. These calculations were followed up with experimental testing on two pipelines.

To test the pipeline as a conductor, the signal was injected onto the pipeline with respect to earth ground. Figure 10 shows the test setup at the UMR pipeline loop. For each frequency, the transmitted signal generator voltage is measured and the received voltage at the other end of the pipeline is measured. The received signal strength (volts) divided by the transmitted signal strength (volts) is the gain of the transmission system (pipeline, in this case).

$$
\text { Gain }=(\text { Received signal amplitude }) /(\text { Transmitted signal amplitude })=v_{o} / v_{i}
$$

The gain is normally reported in $\mathrm{dB}$,

$$
\text { Gain }(\mathrm{dB})=20 \log _{10}\left(v_{o} / v_{i}\right)
$$

A negative gain represents a transmission loss.

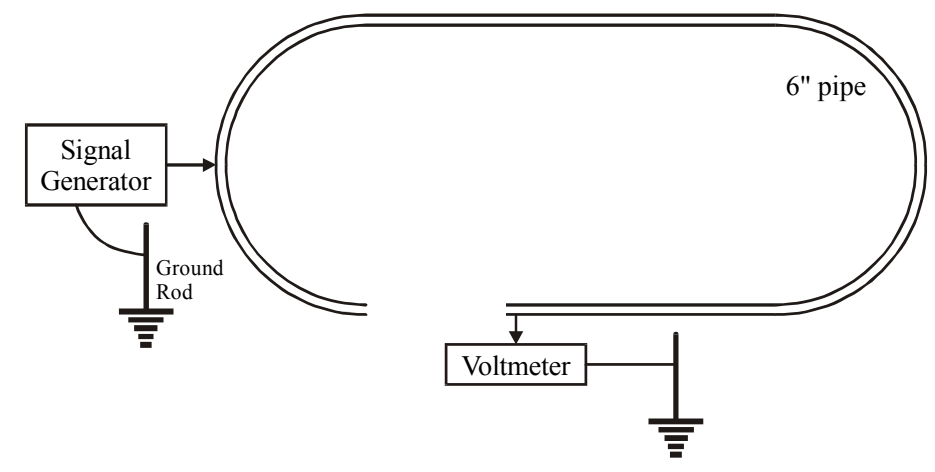

Figure 10. Pipeline as conductor test setup. 


\section{Test Pipelines}

A small scale gas pipeline was constructed for initial tests at the UMR Experimental Mine property. The pipeline is an oval approximately 95 feet long and 40 feet wide (Figure 11). with a 15 foot gap along one side. The pipeline length is approximately 250 feet and the pipes are 6 inch ID steel.

The Battelle Pipeline Simulation Facility (PSF) 24-inch pipeline flow loop is located in West Jefferson, Ohio and shown in Figure 12 (Nestleroth, 1995). For the testing described in this report, a 40 -foot section was removed to allow access in order to place antennas into the pipeline loop. For these tests, the pipeline length was 4721 feet.

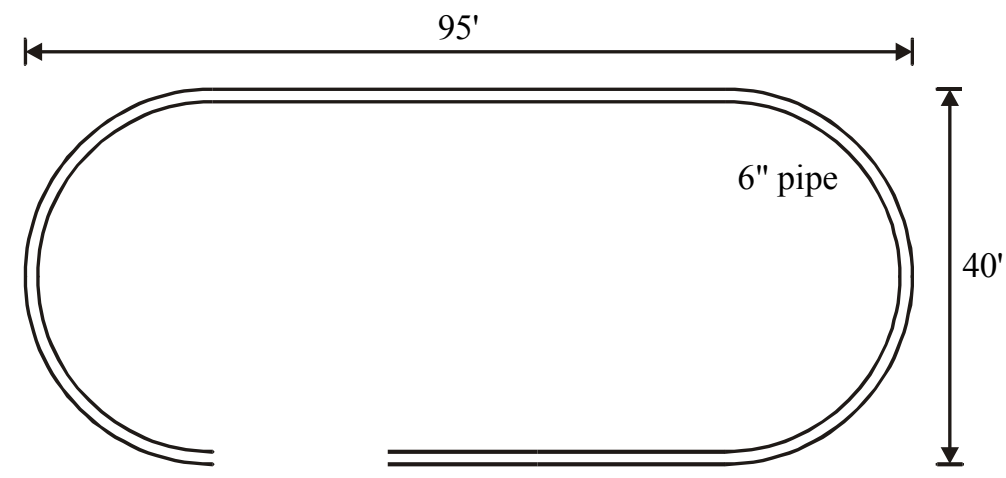

Figure 11. UMR pipeline loop.

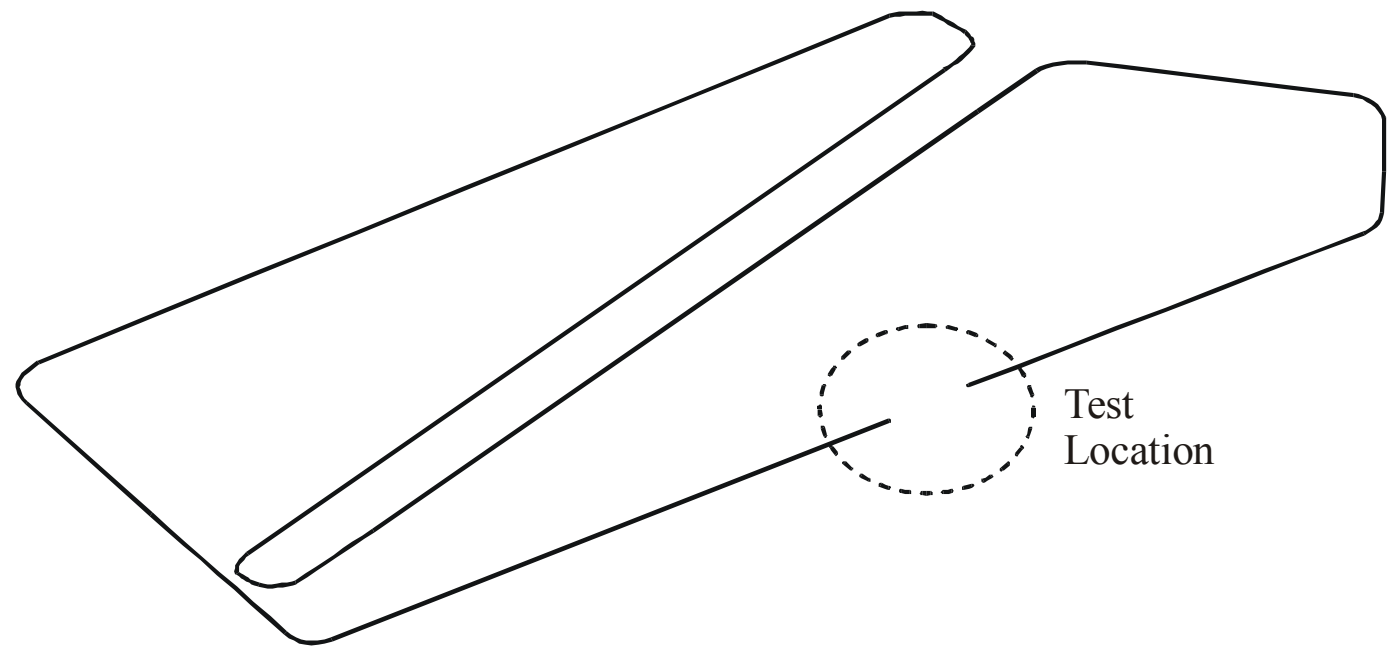

Figure 12. Battelle pipeline simulation facility. 


\section{Communication Protocol}

The main characteristics of the communication protocol were:

1. Routes should be obtained on an on-demand basis (reactive) to insure that the percentage of the bandwidth used for routing control is minimized.

2. Limit the packet overhead.

3. Quickly detect errors in transmission.

4. Quickly update the routing table in the event of a broken link.

5. The routing protocol should be scalable to allow expanding the network.

6. Mobility is not a major concern because the sensors will be stationary. However, the topology is not constant and needs to be updated whenever a change occurs due to a nodes' failure.

7. The routing protocol should guarantee the generation of loop free routes.

The communication network was divided into subnets. Each subnet consisted of a group of sensors that communicated with a central node. The central node was responsible of collecting, filtering, encrypting and forwarding the information to neighboring sensor nodes.

A combination of two routing protocols may be used to achieve a reliable communication system; the first routing protocol will be used to route data between sensors and the central node. The second routing protocol will be used to route packets between central nodes along the pipeline.

The sensors communicate with the central node of their subnet; they are embedded inside the pipeline but shown externally in Figure 13 for illustration purposes. Each central node communicates with other central nodes using the wireless media of the pipeline.

In the following paragraphs, the general characteristics of a suitable protocol are discussed. This project examined the following protocols: Optimized Link State Routing (OLSR), Dynamic Source Routing (DSR), and Ad hoc On-demand Distance Vector (AODV). The results of this study are reported in the Results and Discussion section.

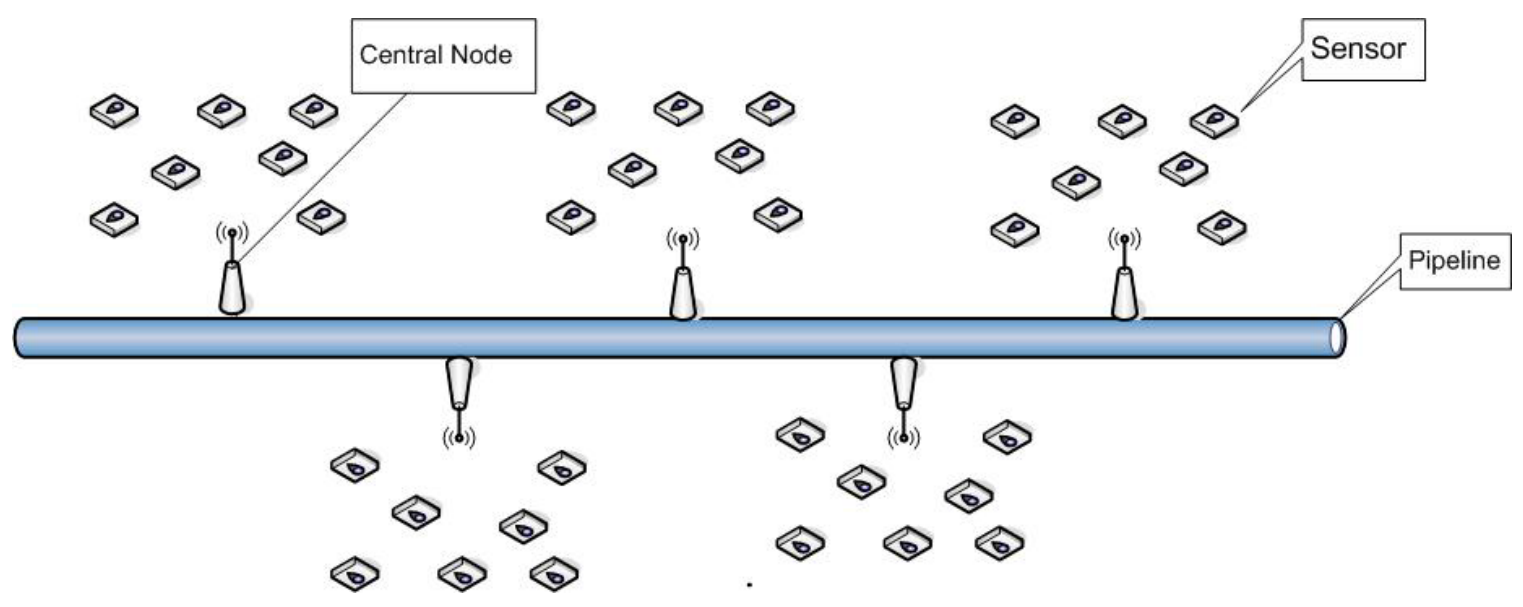

Figure 13. Communication network. 


\section{$\underline{\text { Routing between central nodes: }}$}

The communication between central nodes may adopt several routing options:

1. Static routing: In this routing configuration, each node maintains a table of all the central nodes on the network and the path needed to be taken to send data to a specific destination on the network.

2. Dynamic Routing: Uses a routing protocol in which paths are dynamically updated. This usually results in extra delays in route discovery.

Some of the metrics to be considered in route selection:

- Hop Count: A hop count metric counts router hops.

- Load: This metric reflects the amount of traffic utilizing the links along the path. The best path is the one with the lowest load.

- Delay: Measure of the time a packet takes to traverse a route. A routing protocol using delay as a metric would choose the path with the least delay as the best path.

- Reliability: Measures the likelihood that the link will fail in some way. For example, the number of times a link has failed or the number of errors it has received within a certain time period can be indicative of how reliable the connection is. The path with highest reliability would be selected as best.

\section{$\underline{\text { Routing between nodes within a subnet: }}$}

The sensors on the pipeline will collect the data and pass it to the central node. One way to achieve this is to allow each sensor to transmit directly to the central node. However, this will consume the energy of the sensors resulting in premature failure. Furthermore, the central node will experience a high percentage of collisions which will force the sensors to retransmit and waste more bandwidth and energy.

A better approach is to use the sensor both as host and router. Each sensor is responsible of generating readings for the monitoring system in addition to routing packets from other sensors to the central node.

The routing process starts when a source node has data to be sent to the central node. The source sends a broadcast to all its neighbors containing the (source address, destination address, broadcast ID, hop count). We will call this broadcast the Path Request (PREQ).

The (source address, broadcast ID) are used to uniquely identify the PREQ packet. Whenever the source node issues a new broadcast, it increments the broadcast ID to avoid creating routing loops.

When a node receives a PREQ packet, it checks its routing tables for route information. If the information is available, it sends back a Path Reply (PREP), otherwise it will increment the hop count in the PREQ and rebroadcast it to its neighbors. Each node should store the node ID that transmitted the PREQ in its routing table.

Since a broadcast is used to pass the packets, a node may possibly receive two copies of the PREQ. Nevertheless, by checking the (source address, broadcast ID) combination, it will be able to determine the duplication and drop the extra packet. 
Ultimately, the PREQ will reach the destination or a node that has a current route to the destination. PREP will be sent back in the reverse direction since each intermediate node holds information of the node that initially sent the broadcast to it. The PREP will contain (source address, destination address, hop count). Each intermediate node will store the information in the PREP header to enable it to generate a forward path in addition to the reverse path previously generated.

\section{$\underline{\text { Route Maintenance: }}$}

In normal operation, the routing tables will hold constant for considerably long periods of time because the sensors are stationary. When a sensor fails, it will no longer be able to act as a router for its neighboring nodes and as a result the process of route discovery must be resumed with new PREQ and PREP packets.

\section{$\underline{\text { Security }}$}

Encrypting the payload of data packets sent between the central nodes is an essential part of the communication requirements. More provision should be given to authentication (ensuring that a packet contents are from an actual node on the network and that the contents were not changed).

If an intruder joins the network, he/she may be able to prompt false alarms by sending incorrect information to central nodes. As a result, the whole system may be forced to shut down.

To counter such an attack, public/private key encryption may be used for the authentication of the nodes. The source node would encrypt the data using its private key (not known to others) and the receiver would decrypt it using the public key of the source node.

Another way is to append a Message Authentication Code (MAC) to the packet to guarantee that the contents of the packet were not changed. The source starts by appending a MAC to the packet that is a function of the payload. When the receiver obtains the packet, it recalculates the MAC using the payload of the packet and compares it with the one it originally received. If they match, that means that the contents have not been tampered, otherwise it should discard the packet due to the fact that the contents can not be authenticated. 


\section{Results and Discussion}

\section{Pipeline as Waveguide}

This section presents the results for the overall steps that assessed the feasibility of wireless communication in a pipeline:

1. Experimentally verify the attenuation of electromagnetic waves in a pipeline.

2. Experimentally measure the transmission loss of a 6" pipeline at UMR and a 24" pipeline at Battelle.

3. Use commercial wireless modems to verify that the test pipelines can support wireless communication with commercial-off-the-shelf (COTS) equipment.

\section{Verify attenuation of electromagnetic waves in a pipeline.}

\section{Transmission Line Method}

These tests were done Feb. - Mar., 2003. Through the Time-domain reflectometer (TDR) results as shown in Figure 14, we can see that the characteristic impedance of the pipe is set up to be about $54 \mathrm{ohms}$. Most variations are shown at the two ends with the core shape structures. The highest impedance was $54 \mathrm{ohms}$, the lowest $44 \mathrm{ohms}$. The characteristic impedance of the whole structure was kept within about a 10 percent variation around $50 \mathrm{ohms}$.

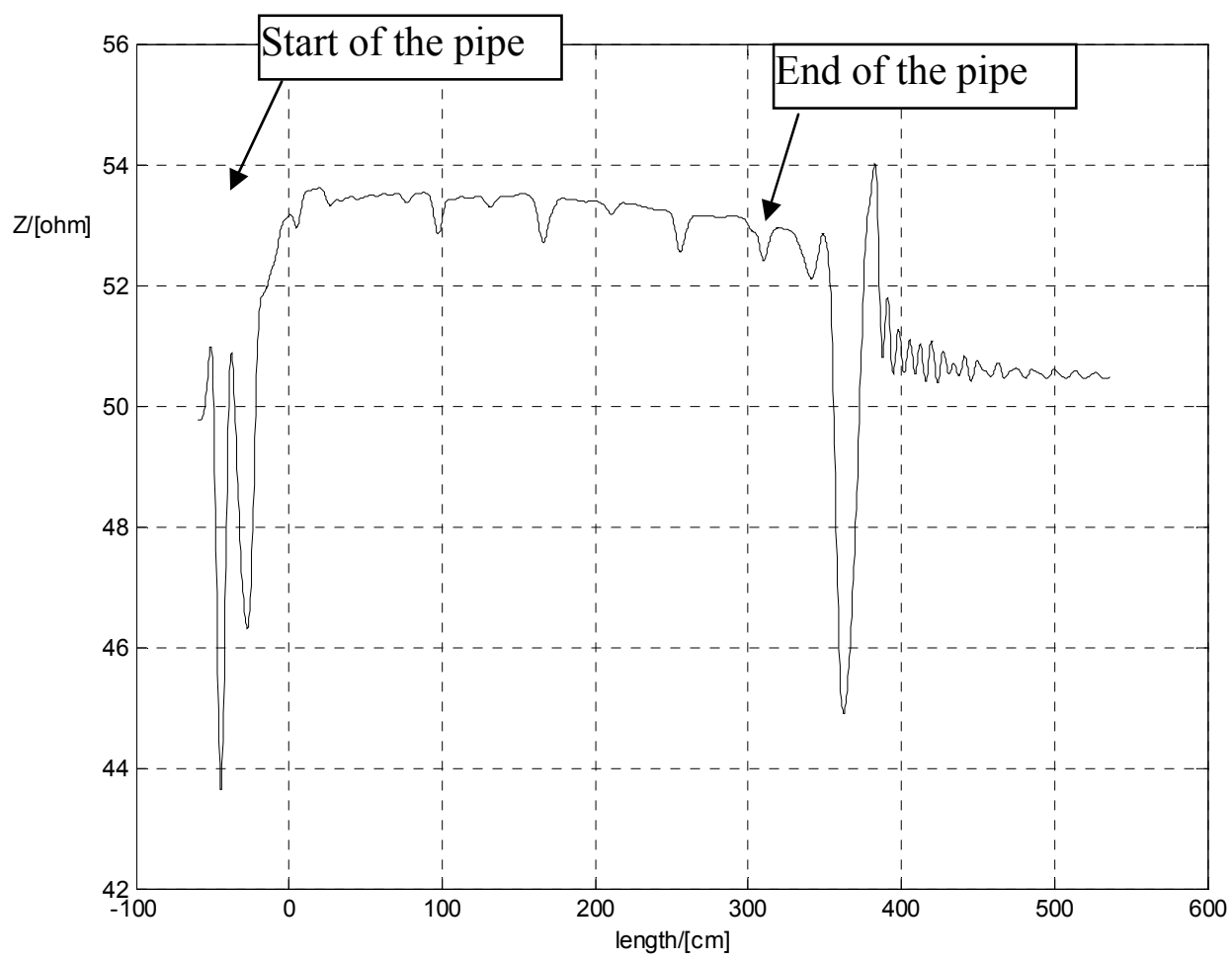

Figure 14: Characteristic impedance through the pipe 
The measurement result of attenuation of the pipe is shown in Figure 15. The oscillation on the original waveform is related to residence frequency of the pipe, which is about $39 \mathrm{MHz}$. The smoothed curve shows clearly an increase of the attenuation of the pipe with the frequency. Figure 15 indicated a $3 \mathrm{~dB}$ drop for frequencies over $3 \mathrm{Ghz}$. This is quite a large loss for such a small pipe length (3.5 meters).

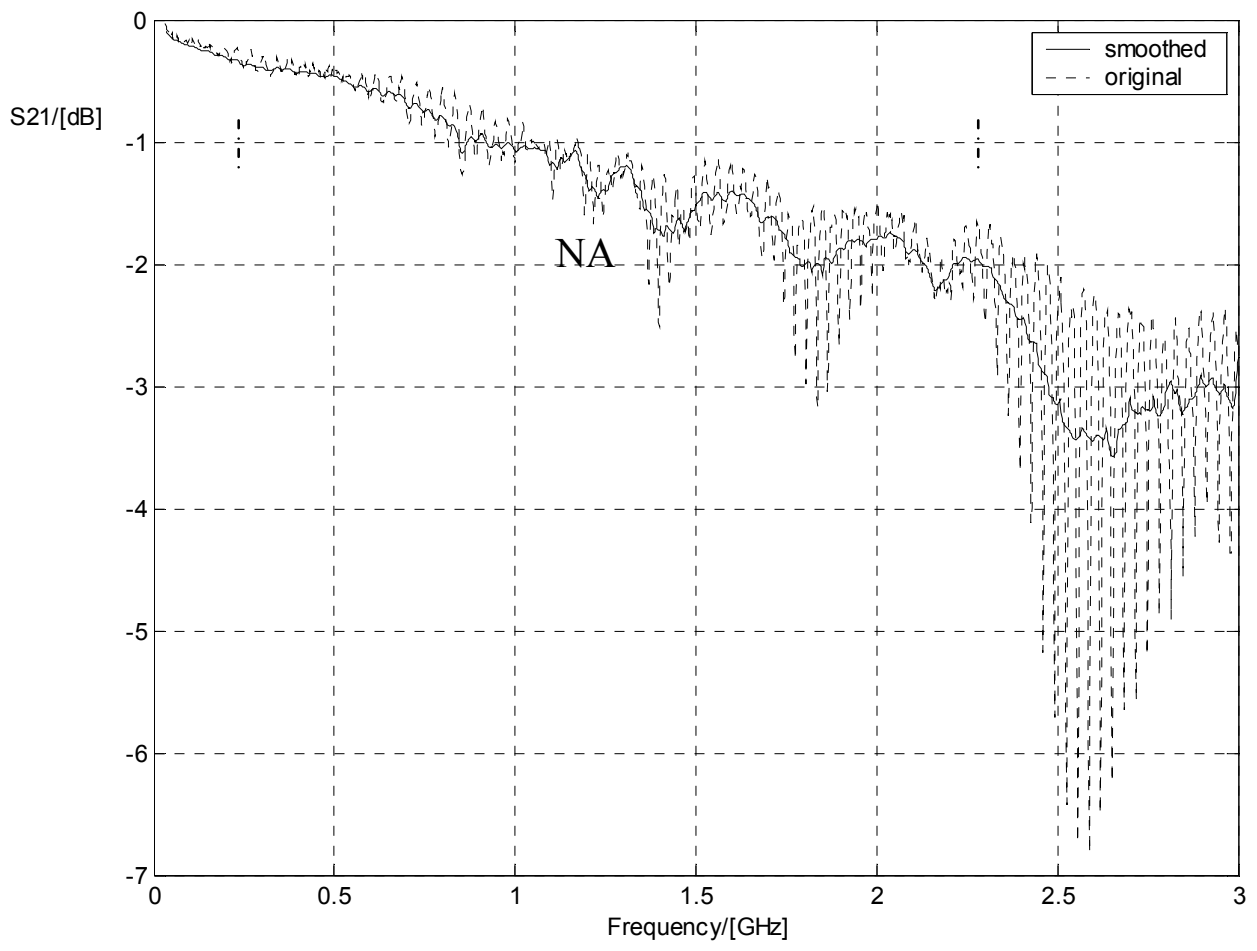

Figure 15:Attenuation of the pipe

\section{Resonance Cavity Method}

These tests were setup and performed May - Oct., 2003. For the Q-factor measurement, the probe length of $0.75 \mathrm{~m}$ was long enough to excite the $\mathrm{TE}_{111}$ mode. Figure 16 shows the return loss, $\mathrm{S}_{11}$, with the probe in the position shown in Figure 5. Given the pipe's inner diameter $2 \mathrm{a}=0.15738 \mathrm{~m}$ and length $\mathrm{d}=3.5 \mathrm{~m}$, the resonant frequency of the dominant $\mathrm{TE}_{111}$ mode was calculated as $1.1179 \mathrm{GHz}$. Actually, the first peak occurred at $1.1071 \mathrm{GHz}$. Actually, the accuracy of the pipe's inner diameter has a significant influence on calculating the resonant frequencies. A diameter with statistical meaning should be used in the calculation. However, there was no way to have a precise measurement of the pipe's inner diameter all over its length. However, a diameter of $0.15738 \mathrm{~m}$ was measured at the ends of the pipe. However, a diameter of $0.15920 \mathrm{~m}$ matched with the measured peak frequency. 
The $\left(1-\left|\Gamma\left(f_{0}\right)^{2}\right|\right)$ plot of the pipe from $1.1 \mathrm{GHz}$ to $1.3 \mathrm{GHz}$ is shown in Figure 17.

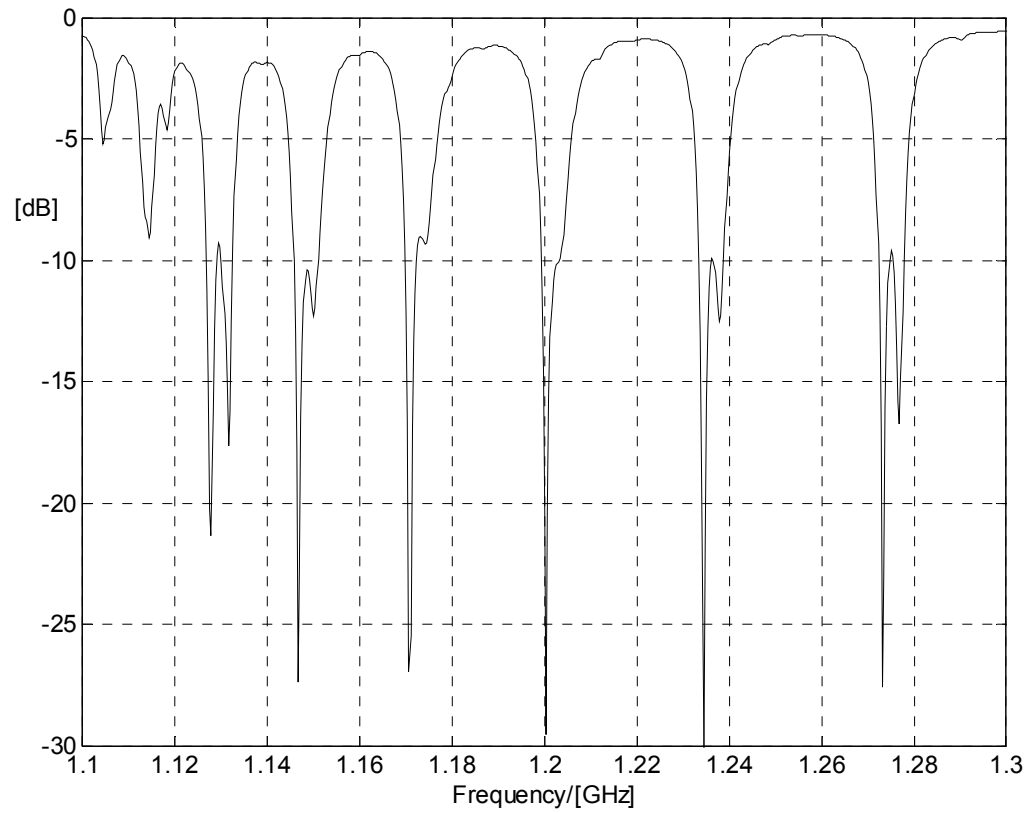

Figure 16. $S_{11}$ of the pipe from $1.1 \mathrm{GHz}$ to $1.3 \mathrm{GHz}$.

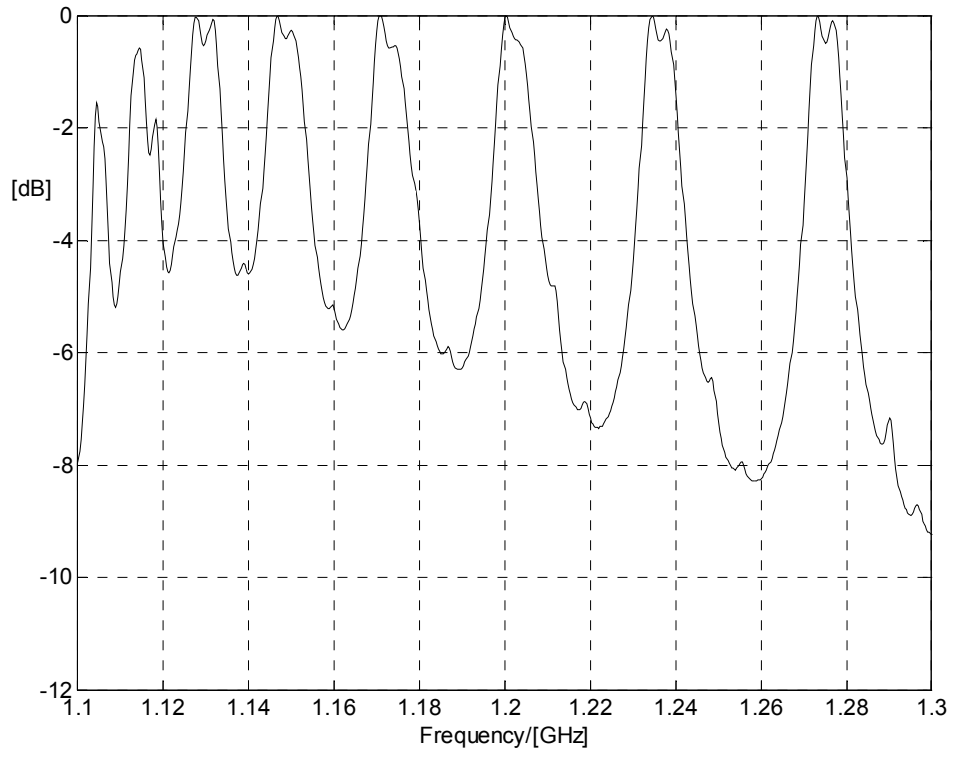

Figure 17. Q-factor measurement of the pipe 
Figure 16 shows that, at $1.2 \mathrm{GHz}$, the $11^{\text {th }}$ resonant $\mathrm{TE}_{11 l}$ mode has a peak about $30 \mathrm{~dB}$. So it is at about critical coupling. The $\left(1-\left|\Gamma\left(f_{0}\right)^{2}\right|\right)$ plot in Figure 17 shows its $3 \mathrm{~dB}$ bandwidth about $0.01 \mathrm{GHz}$. So the loaded Q-factor is calculated as 114 and the unloaded Q-factor is 228 .

The conducting loss coefficient of the pipe is calculated as:

$$
\begin{aligned}
& Q=\frac{(k a)^{3} \eta a d}{4\left(P_{m n}^{\prime}\right)^{2} R_{s}} \frac{1-\left(\frac{m}{\left(P_{m n}^{\prime}\right)^{2}}\right.}{\left\{\frac{a d}{2}\left[1+\left(\frac{\beta a m}{\left(P_{m n}^{\prime}\right)^{2}}\right)^{2}\right]+\frac{\left(\beta a^{2}\right)^{2}}{\left(P_{m n}^{\prime}\right)^{2}}\left(1-\left(\frac{m}{\left(P_{m n}^{\prime}\right)^{2}}\right)\right\}\right.} \\
& R_{s}=\sqrt{\frac{\omega \mu}{\sigma_{c}}}
\end{aligned}
$$

where

$$
k=\omega \sqrt{\mu \varepsilon} \text { and } \beta=\sqrt{k^{2}-\frac{\left(P_{m n}^{\prime}\right)^{2}}{a^{2}}} .
$$

Finally, the conducting loss coefficient of the pipe is calculated as $2.8 \times 10^{3} \mathrm{~s} / \mathrm{m}$.

\section{$\underline{\text { Measure transmission loss of pipeline. }}$}

The UMR pipeline loop was used for initial pipeline testing in Nov., 2003. Figure 18 shows the transmission loss of the pipeline measured with the two antennas. The frequency ranges from $1 \mathrm{GHz}$ to $6 \mathrm{GHz}$. The blue curve shows the noise floor of the transmission loss for the two antennas. The other curve shows the transmission loss of the pipeline. Below $4 \mathrm{GHz}$, most of the power from the transmitter antenna was lost through the pipe and very little was received by the receiver antenna. After $4 \mathrm{GHz}$, the transmitted power experienced a continuous increase with increasing frequencies. At $6 \mathrm{GHz}$, it has about $-60 \mathrm{~dB}$ gain and about $20 \mathrm{~dB}$ above the noise floor. 


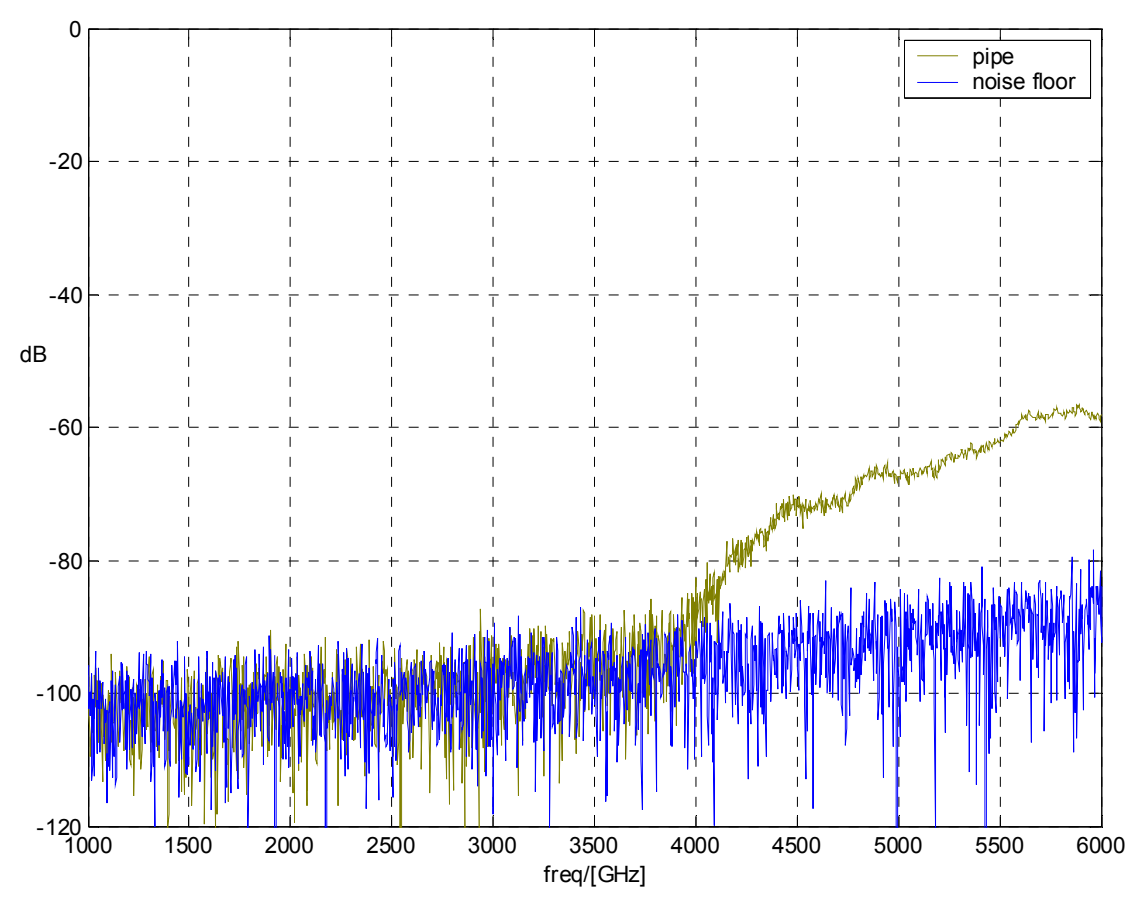

Figure 18. Transmission loss of UMR pipeline.

The Battelle pipeline was used for tests in Sept. and Nov., 2004. The test setup with the Battelle pipeline was similar to that of the UMR pipeline. The pipeline was large enough to completely contain the antenna. So, each antenna was placed in the pipeline end in the approximate center of the pipe and the pipeline ends were completely sealed with aluminum foil. Electromagnetic absorbing foam was also placed outside the pipe end to further reduce signal transmission across the 40-foot air gap. The transmission loss of the pipeline showed a small peak (at $-62 \mathrm{~dB}$ ) at around 1.0 GHz (Figure 19, black trace). The other traces on the graph were obtained as follows:

Baseline thru (red) - The two antennas were pointed at each other with a gap of a few feet. This trace shows the attenuation was small, as expected.

Antennas in free space (yellow) - The antennas were pointed at each other across the 40 foot air gap. This trace shows the attenuation of the signal in a (relatively) small distance through air.

Noise floor (black) - The antennas pointed in opposite directions. This trace shows the amount of noise in the system, which is the signal at the receiver that does not come from the transmitter.

Valve shutdown (green) - The transmission loss of the pipeline when an intermediate gate valve was closed. It should be nearly identical to the noise floor since no signal should be able to penetrate the closed valve. 


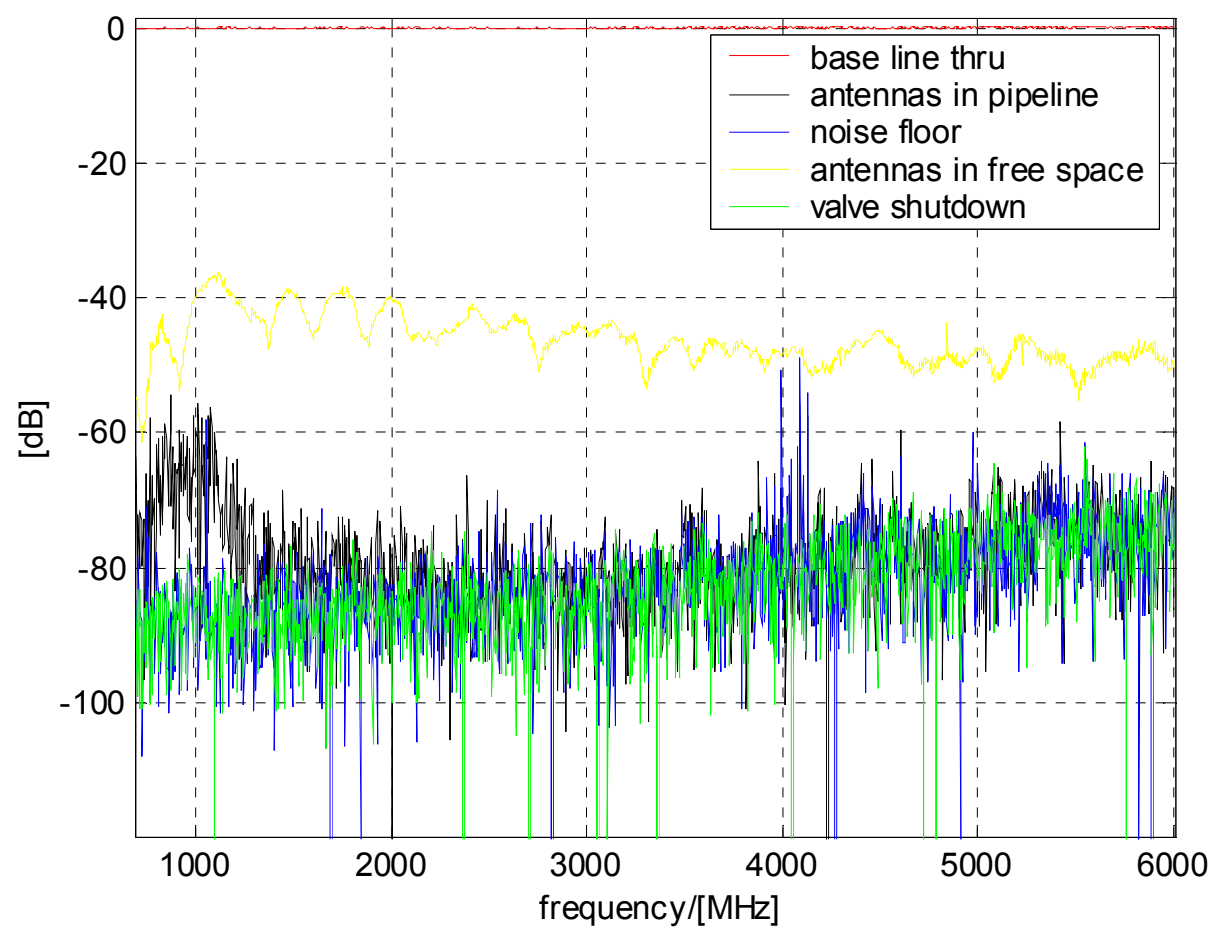

Figure 19. Transmission loss of Battelle pipeline.

\section{Use commercial wireless modems on test pipelines.}

The commercial $802.11 \mathrm{~b}$ modems were tested with the both pipelines. For the UMR pipeline, the modems used the 6" omni-directional whip antenna oriented vertically. In these tests, the signal strength indicated by 3 LED indicators on the modem could not be ascertained since the modems were both inside the pipeline and the pipeline ends sealed. However, the two PLC's successfully communicated after about one minute (The modems required about one minute to successfully negotiate and start the TKIP encryption that they were configured for). "Successful communication" means the two PLC's were exchanging messages, indicated by a flashing O:2/00 discrete output on both PLC's. This test was performed in Nov., 2003.

For the Batelle pipeline, communication was successful when one of the modems used the horn antenna and the other used the dish/feed antenna. Communication was also successful with $8 \mathrm{dBi}$ collinear array antennas (about 18" long) oriented vertically. For both of these situations, the modems indicated good signal strength (all three signal-strength LED's on). Communication was not successful when one of the collinear array antennas was oriented parallel with the pipe, or when one of the collinear array antennas was replaced with a $2 \mathrm{dBi}$ whip antenna (about 6" long). In this case, the PLC's had not started exchanging messages after waiting 5 minutes. Also, all three signal-strength LED's on the modems were off, indicated that neither modem was receiving a signal from the other modem. This test was performed in Nov., 2004 
To verify that the signals were not leaking out of any possible openings in the foil covering the pipeline ends, a pipeline gate valve was closed. Communication between the modems abruptly ceased. When the valve was opened, communication resumed after the minute that seems to be required to re-establish the TKIP encrypted communication.

\section{Discussion of Results.}

Initial testing of a 3.5-meter section of pipe showed an attenuation of $3 \mathrm{~dB}$, which is equivalent to half of the signal power being lost, which is a large loss. However, when the pipe is set up as a resonating cavity, the loss for certain frequencies was quite low, indicating the possibility that the pipeline could transmit frequencies of a few $\mathrm{GHz}$.

Following the initial analysis, the attenuation characteristics of actual pipelines was measured. Both the UMR pipeline and Battelle pipelines showed significant attenuation, but the attenuation was not large enough to prevent commercial wireless radios from effectively working. For both of these pipelines, a point-to-point $802.11 \mathrm{~b}$ wireless link was set up between two Ethernet PLCs and messages were transmitted between the PLCs, evidenced by a flashing indicator on the PLCs. The signal strength was not assessed on the UMR pipeline since the modems were sealed inside the pipeline and the signal-strength indicators were not visible. However, when testing the same type of link on the Battelle pipeline, one of the modems was kept outside so the signal-strength indicators were visible. On the Battelle pipeline wireless link, the signal strength was good (shown by the modem signal-strength indicators) when the radios used the $8 \mathrm{dBi}$ collinear array antennas.

The UMR and Battelle pipelines showed less attenuation for frequencies in the ranges of $5 \mathrm{GHz}$. So, 802.11 a wireless radios should be able to transmit farther than the $802.11 \mathrm{~b}$ radios used for these tests. 


\section{Pipeline as Conductor}

The developed models are described first, followed by the calculation of the expected attenuation of the pipeline at frequencies up to $1 \mathrm{MHz}$. These calculations were followed up with experimental testing on two pipelines.

\section{$\underline{\text { Pipeline Models }}$}

Three separate models were developed for three distinct cases:

1. Pipelines supported above the earth.

2. Pipelines lying on the surface of the earth.

3. Pipelines buried beneath the surface of the earth.

In each case, it was necessary to estimate the series inductance, series resistance, shunt capacitance and shunt conductance. These models were developed from Jan. - June, 2003.

Series Resistance. The series resistance is the same for all three cases. The series resistance is basically the product of the resistivity and the length divided by the cross sectional area. The only complicating factor is the skin effect. The skin effect will be more pronounced for pipelines made of ferroelectric materials and at the higher frequencies used to send control and communications signals. An approximation was used in which the pipe is modeled as a series of concentric shells with shells farther from the surface having lower current densities. The ac resistance was found by adding the losses in all shells and dividing by the square of the RMS current.

Series Inductance. The series inductance is basically the inductance of a single conductor with earth return. As in the case of series resistance, the skin effect was significant and it had a strong influence on the internal inductance. Once again the shell model allowed the inclusion of the skin effect on the internal inductance.

The calculation of the external inductance depended on which of the three cases was being dealt with: above surface, surface or subsurface.

The above surface case is the most straightforward. In this case the influence of the earth was modeled as an image conductor the same depth below the surface as the physical conductor is above the surface. The influence of finite earth conductivity produced an image conductor deeper below the surface than in the case of perfectly conducting earth. The phenomenon was handled using corrections formulated by Carson (1926).

The case of the pipe lying on the earth was handled in essentially the same way. In this case there was no external flux without the corrected return depth.

The case of the pipe buried in the earth was the most challenging. The methodology used to calculate the zero sequence inductance of buried cables was extrapolated to this configuration. This was probably the most challenging problem.

Shunt Conductance. Shunt conductance for pipelines supported above the earth was assumed to be zero. Clearly, this fact makes this class of pipelines the most likely candidates for using the pipeline itself to conduct control and communications signals. 
It was assumed that every pipeline will have some coating to retard corrosion, even if it is only a coat of paint. For pipes lying on the earth, equations for conductance to earth of pipes half buried was used. The presence of the coating on the pipe was handled by treating the coating as a layer of low conductivity earth. Equations for this case are given in Sunde (1968). In a similar fashion for pipelines beneath the surface of the earth, equations from Sunde (1968) text were used.

Shunt Capacitance. Shunt capacitance for pipelines supported above the earth was calculated using conventional formulas for single conductors above earth.

Similarly, for pipelines lying on the surface of the earth, equations for single conductors above the earth were used.

For the case of conductors buried beneath the earth, it was assumed that an insulating coating exists. This assumption allowed the capacitance to be calculated using formulas for coaxial cylinders separated by a dielectric.

\section{Parametric Analysis}

Once the models were fully developed, a variety of cases for different pipeline materials, diameters, wall thicknesses, coatings, earth resisitivities $\left(\rho_{e}\right)$ and frequencies were calculated during summer, 2003. The results of the calculated attenuation versus $\rho_{e}$ and frequency are shown in Figures 20 through 25 for

cast iron pipe, and stainless steel pipe

for above-ground, half-buried, and fully-buried 10 miles of pipe. In these figures, "roe" is the earth resistivity, $\rho_{e}$. Figure 26 shows that the burial height for a half-buried pipe has negligible effect on the attenuation. The pipe diameter and wall thickness also had little effect on the attenuation characteristics. A listing of the program that performed the calculations is in Appendix B.

Examination of Figures 20 through 25 reveals some interesting facts. First, regardless of earth resistivity, the attenuation becomes quite pronounced for frequencies much above $1 \mathrm{kHz}$. Second, attenuation was lower for higher resistivity. This result is contrary to one's intuition since the return current flows through this higher resistance but the decrease in shunt losses which are less when the earth resistivity is larger, is a more important effect. This decrease in shunt losses gives lower attenuation. 


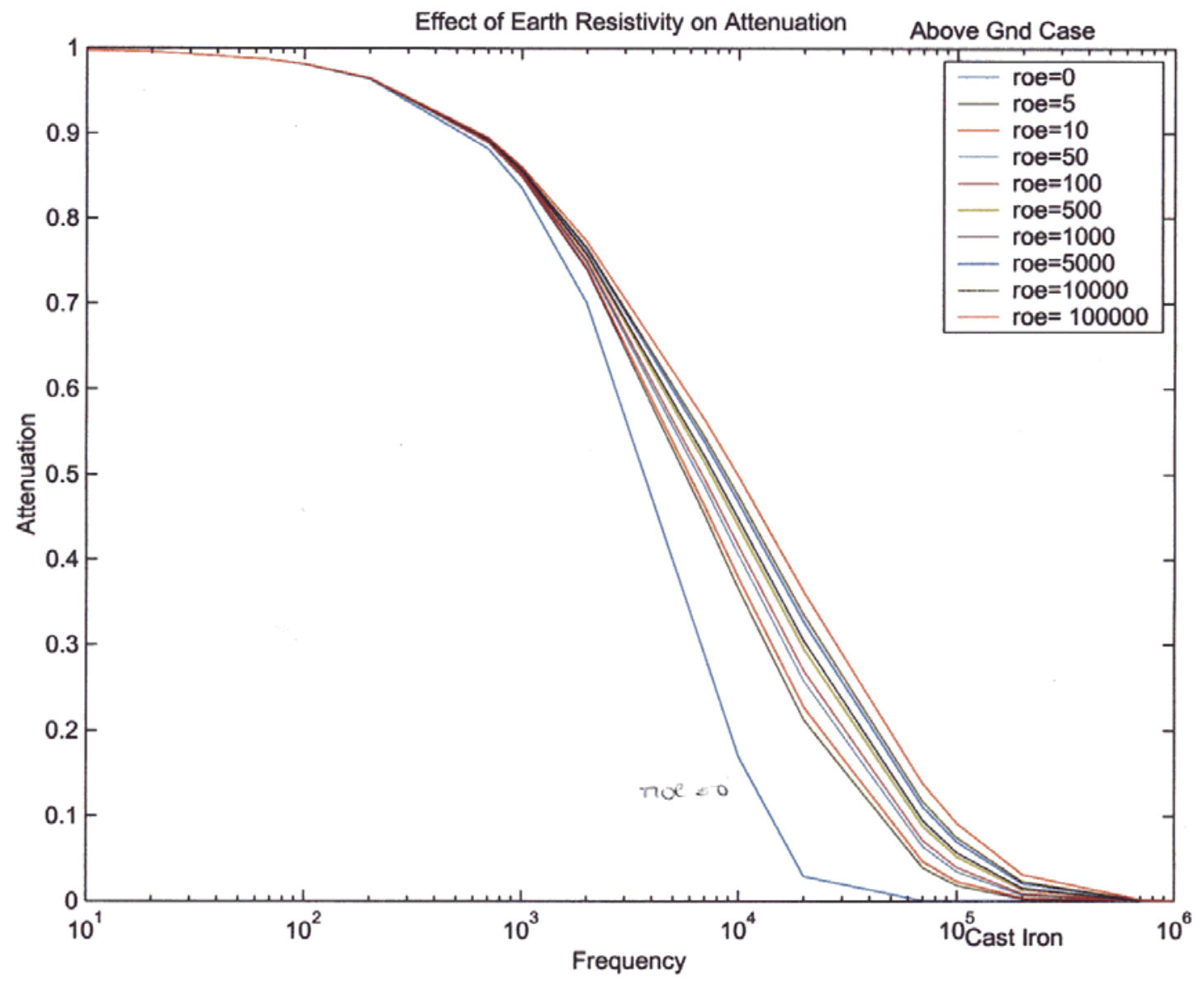

$$
\begin{aligned}
& 10 \text { mile pipeline } \\
& \text { roe is resistivity }
\end{aligned}
$$

Figure 20. Attenuation as a function of frequency: Cast iron pipe, above ground. 


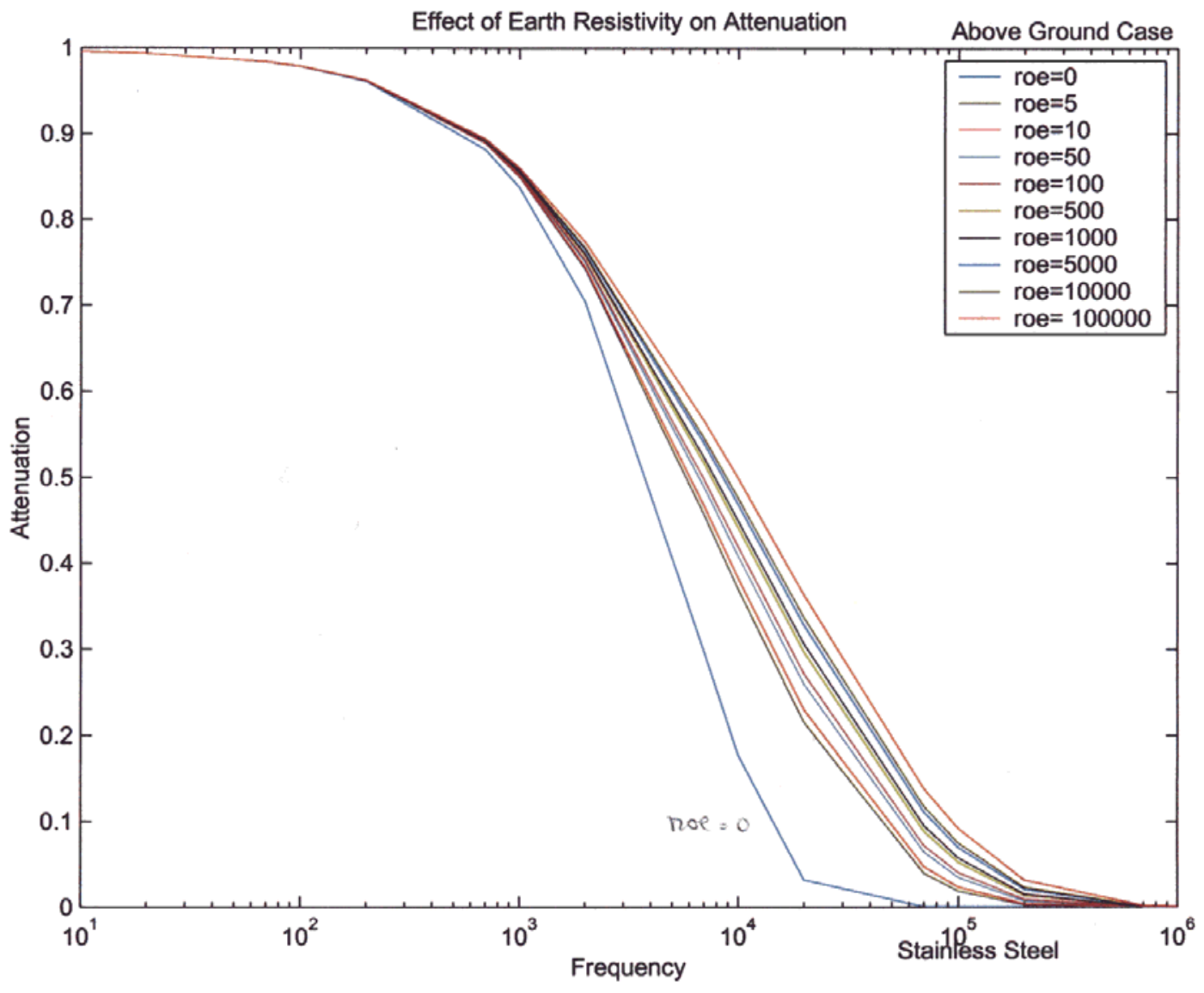

Figure 21. Attenuation as a function of frequency: Stainless steel pipe, above ground. 


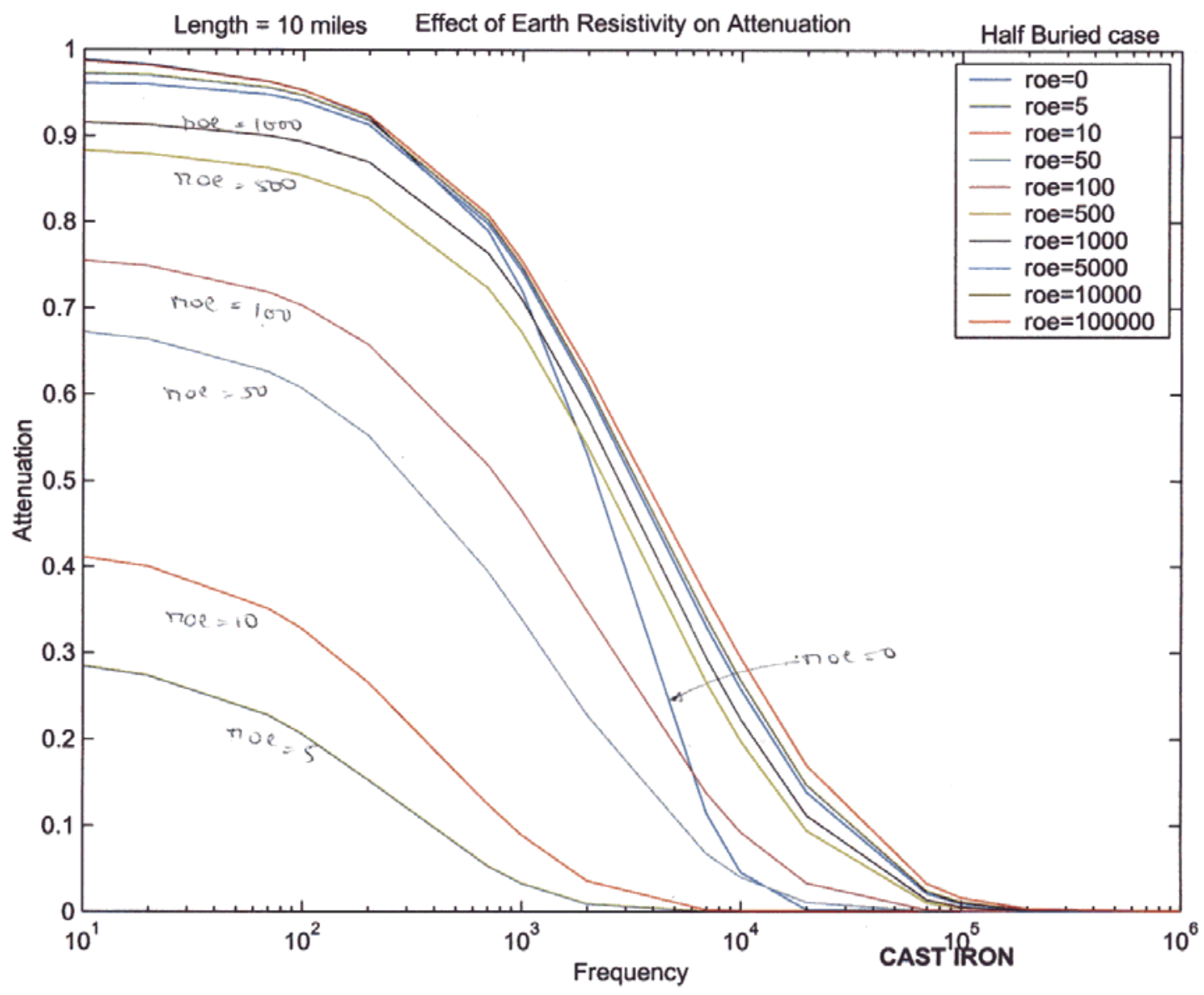

Figure 22. Attenuation as a function of frequency: Cast iron pipe, half-buried. 


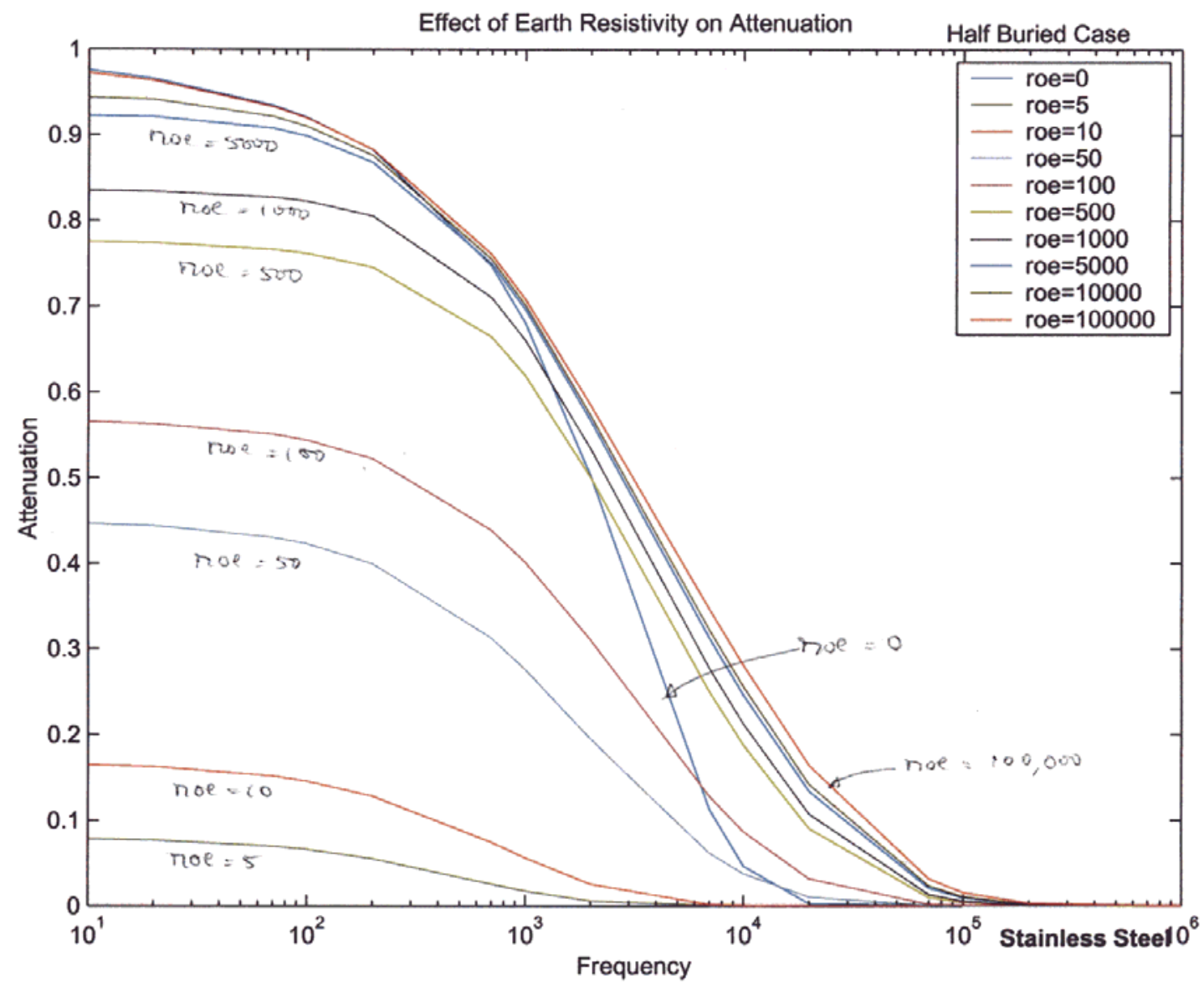

Figure 23. Attenuation as a function of frequency: Stainless steel pipe, half-buried. 


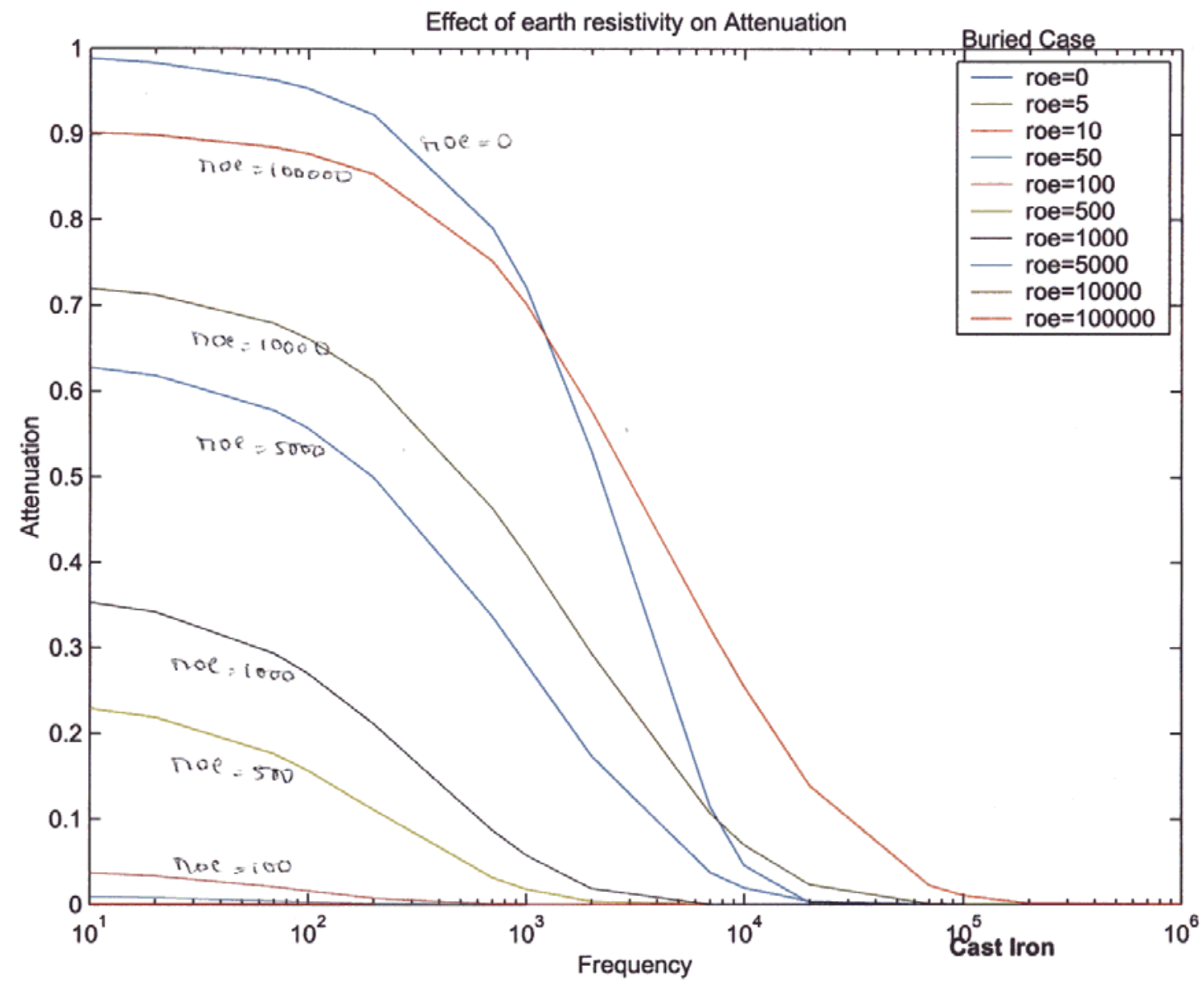

Figure 24. Attenuation as a function of frequency: Cast iron pipe, fully-buried. 


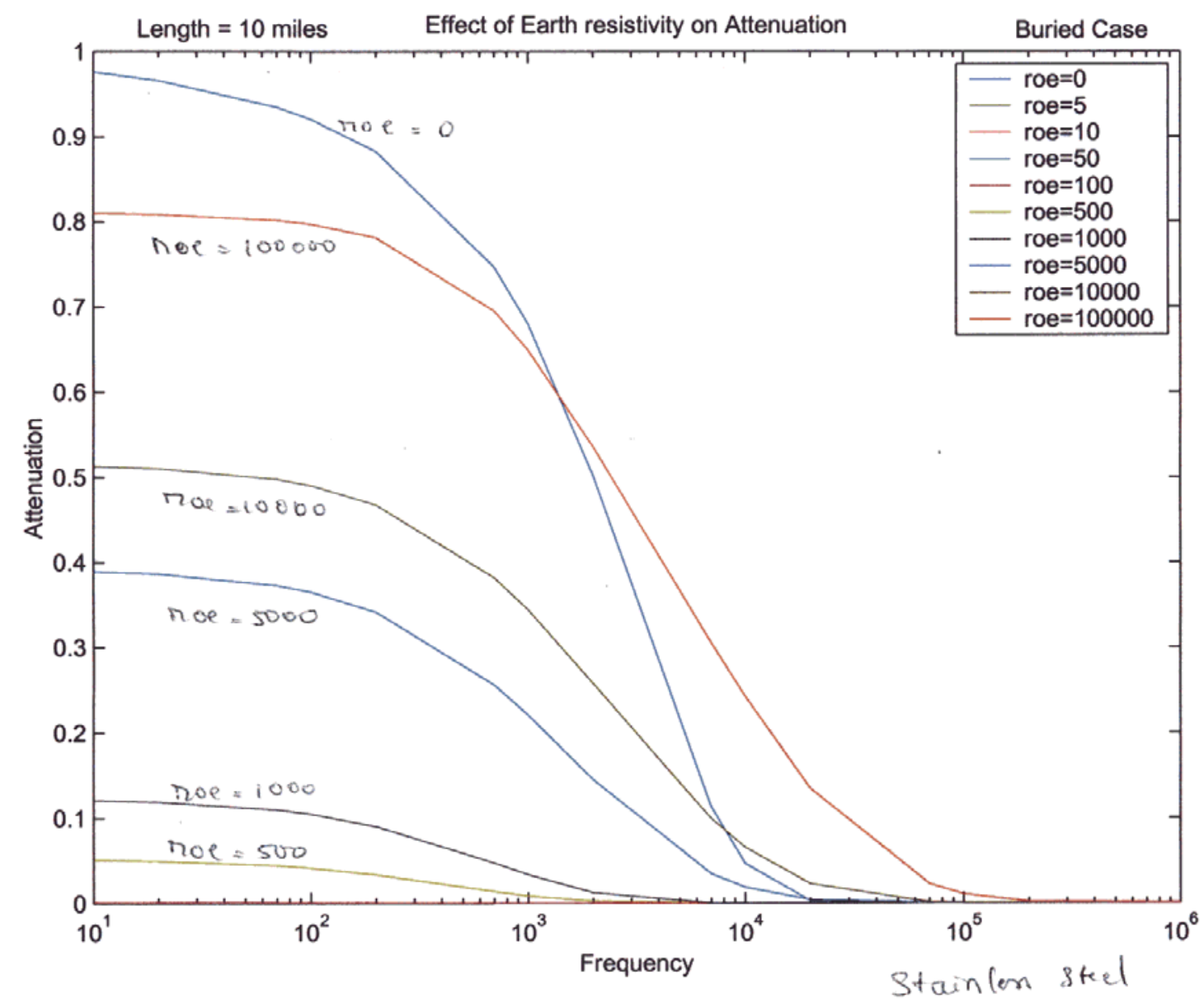

Figure 25. Attenuation as a function of frequency: Stainless steel pipe, fully-buried. 


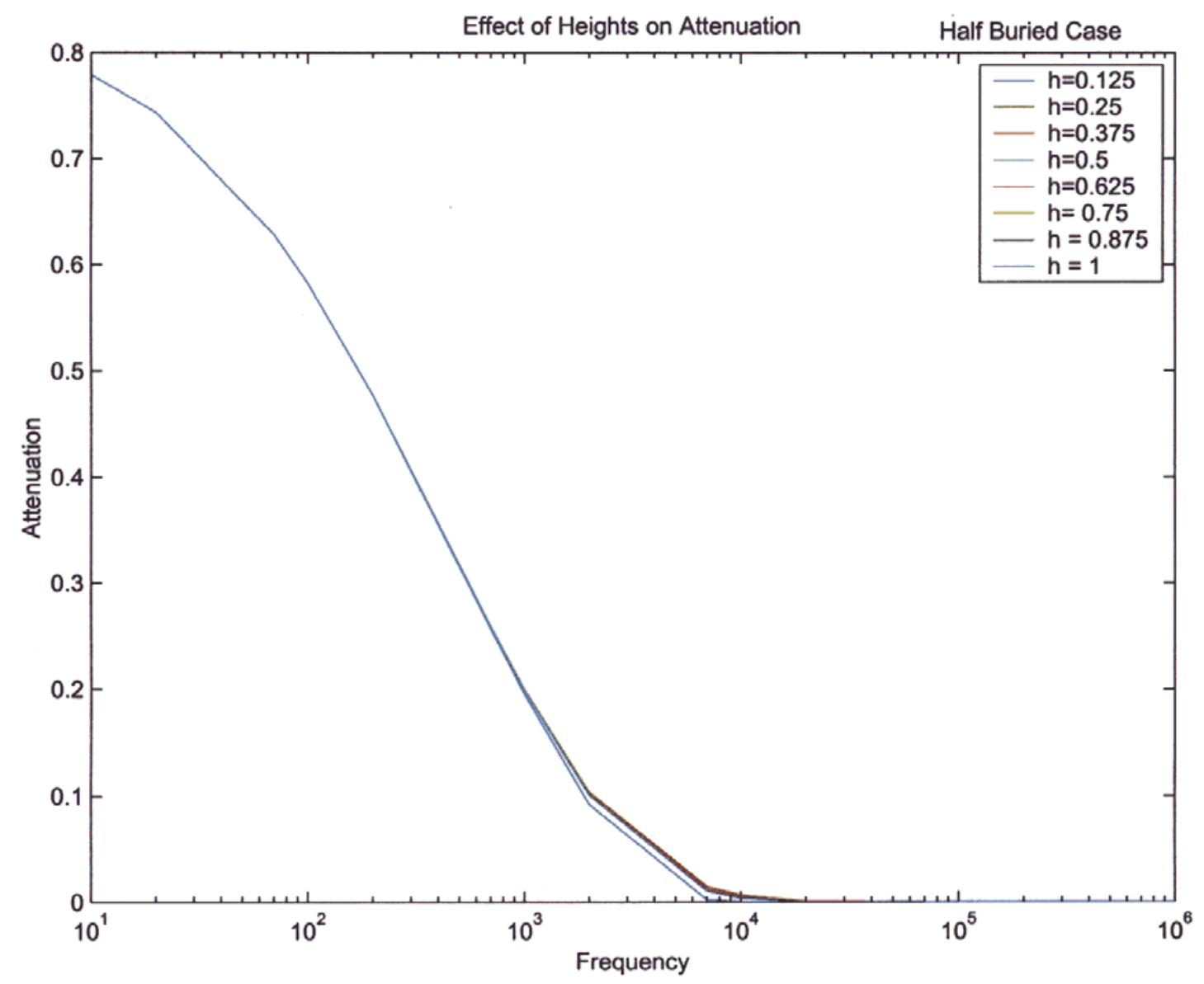

Figure 26. Attenuation as a function of frequency: Effect of burial height (h) on half-buried pipe. 


\section{$\underline{\text { Attenuation Measurements }}$}

To test the pipeline as a conductor, the signal was injected onto the pipeline with respect to earth ground. Figure 10 shows the test setup at the UMR pipeline loop in March, 2004. The UMR pipeline showed little attenuation (Figure 27).

The tests were repeated with the Battelle pipeline loop in November, 2004. The cathodic protection was disconnected from the pipe and the pipe exhibited a -0.5 volt potential with respect to earth. In this case, the signal was imposed on the pipeline with an audio amplifier connected to the signal generator. The audio amplifier provided a much-needed current boost to the signal. Two sets of tests were performed. First, the signal was measured at a point 40 feet away from the end of the pipe where the sine wave is injected. In order to verify that signals were being imposed on the pipeline, the gain at only a few frequencies was determined at this point (Figure 27). Since a signal was being received larger than the noise floor of about $150 \mathrm{mV}$, the response at the other end of the pipe (4721 feet away) was measured. With no signal imposed on the pipeline, the output voltmeter measured $200 \mathrm{mV}$ rms noise on the pipe. The measured source and output voltages for the various frequencies were as follows:

\begin{tabular}{|c|c|c|}
\hline Freq. (kHz). & Source $(\mathrm{V}, \mathrm{rms})$ & Output (V,rms \\
\hline 0.10 & 1.06 & $0.196 \mathrm{mV}$ \\
\hline 1.0 & 5.30 & $0.205 \mathrm{mV}$ \\
\hline 1.6 & 5.66 & $0.200 \mathrm{mV}$ \\
\hline 2.5 & 6.36 & $0.210 \mathrm{mV}$ \\
\hline 4.0 & 6.72 & $0.237 \mathrm{mV}$ \\
\hline 6.3 & 7.07 & $0.287 \mathrm{mV}$ \\
\hline 10.0 & 7.07 & $0.375 \mathrm{mV}$ \\
\hline 16.0 & 4.24 & $0.449 \mathrm{mV}$ \\
\hline 25.0 & 5.30 & $0.501 \mathrm{mV}$ \\
\hline 40.0 & 5.30 & $0.565 \mathrm{mV}$ \\
\hline 63.0 & 4.24 & $0.447 \mathrm{mV}$ \\
\hline 100.0 & 3.54 & $0.275 \mathrm{mV}$ \\
\hline 160.0 & signal genera & mplifier not wor \\
\hline
\end{tabular}

The gain at the frequencies is shown in Figure 27. Note that even though the gain at $0.1 \mathrm{kHz}$ looks good, the received signal was not higher than the noise floor. The tests show that this pipeline can effectively transmit a signal in the range of $10-60 \mathrm{kHz}$. 


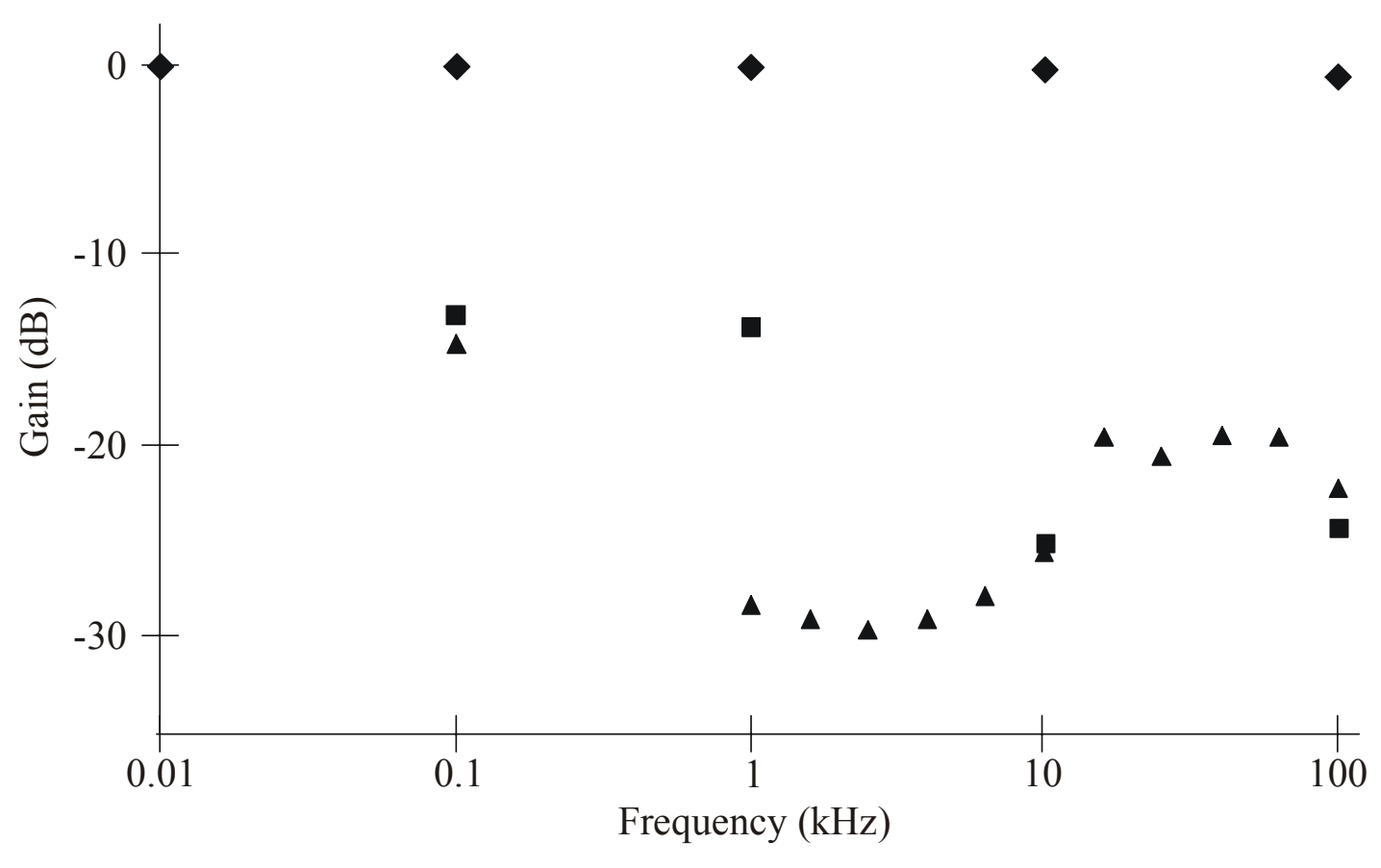

UMR $260 \mathrm{ft}$ - Battelle $40 \mathrm{ft}$. $\quad$ Battelle $4721 \mathrm{ft}$.

Figure 27. Signal gain as a function of frequency.

\section{Discussion of Results.}

A parametric analysis of above-ground, half-buried, and fully-buried 10 miles of pipe showed that the pipe diameter and wall thickness had negligible affect on the attenuation. Also, the burial height for a half-buried pipe had a negligible effect on the attenuation. Regardless of earth resistivity, the attenuation becomes quite pronounced for frequencies much above $1 \mathrm{kHz}$. Surprising, the attenuation was lower for higher earth resistivity.

Following the initial analysis, the attenuation characteristics of actual pipelines were measured. The UMR pipeline showed little attenuation, which was not surprising since the pipeline had very little contact with the earth. The Battelle pipeline (which was mostly buried) showed significant attenuation for a distance of 0.9 miles for frequencies in the range of $10 \mathrm{kHz}$ to $60 \mathrm{kHz}$. These frequencies are in the range of commercial audio telephone modems. The attenuation was not large enough to prevent commercial telephone modems from establishing communications (with an appropriate amplifier on the receiver). 


\section{Communication Protocol}

Studies were performed to analyze and compare the Optimized Link State Routing (OLSR), the Dynamic Source Routing (DSR), and the Ad hoc On-demand Distance Vector (AODV) routing protocols.

Using a network simulator, we simulated a large wireless network without the constraints of a physical network. Using this simulator, each protocol was tested with the exact same random scenario, thus, the difference between protocols is based purely on their implementation. The protocols were analyzed using a 50-node network with varying node speeds and pause times. While the application here would likely utilize fixed nodes, we did not consider mobility as well. The network size was then increased to a 100-node network to determine how the protocols scale to a larger network size. Finally, an analysis of node distance was performed to see if it has any bearing on the data collected.

From the results that were obtained by these simulations, OLSR performed the best for the large scale network. While the data delivery rate was not quite as high as one would hope, especially at higher node speeds, delivery rate probably could be increased with a change of the OLSR parameters to allow more frequent neighbor sensing and multipoint relay set broadcast. With a large or dense network with a dynamic topology, OLSR seems to be the better choice, especially if the data is needed in near real-time applications. With small networks or networks with low node mobility, DSR would probably be a better choice because of its high data delivery rate, although a user must figure in the added path delay in choosing DSR. For high node mobility and a small network size, DSR or AODV are a better choice only if the amount of control overhead, delay, and path length are adequate otherwise, OLSR would be a better choice. For a situation in which the network size or node mobility might be unknown or vary, OLSR seems to be the better choice. 


\section{Conclusion}

The goal of this project was to investigate the use of natural gas pipeline as a wireless waveguide and as a conductor to support remote communications. The experimental results presented in this report indicate that both methods of transmitting signals are feasible.

A pipeline can act as a waveguide to frequencies in the few $\mathrm{GHz}$ range. In this project, the attenuation of the signal through a pipeline of up to 0.9 miles long was not too severe for frequencies up to $6 \mathrm{GHz}$ (the highest frequency supported by our instrumentation). Commercial $802.11 \mathrm{~b}(2.4 \mathrm{GHz})$ modems with medium-gain antennas were able to establish a wireless communication link through a 0.9 -mile 24 -inch pipeline. We did not test the communication through longer pipelines. However, since the modems indicated good signal strength at this distance, we expect that wireless communications can be supported for at least 2 miles. The radios had a limit of 200 milliwatts of transmitted power. Inserting an amplifier between the radio and the antenna should boost this range even further. Also, since both test pipelines showed less attenuation for frequencies in the ranges of $5 \mathrm{GHz}, 802.11 \mathrm{a}$ wireless radios should be able to transmit farther than the $802.11 \mathrm{~b}$ radios used for these tests.

A pipeline can also support direct signal injection of a signal with a frequency of a few $\mathrm{kHz}$. A parametric theoretical analysis of a 10-mile pipeline showed that the attenuation becomes quite pronounced for frequencies much above $1 \mathrm{kHz}$. The attenuation of a mostly-buried 0.9mile pipeline showed significant attenuation for a distance of 0.9 miles for frequencies in the range of $10 \mathrm{kHz}$ to $60 \mathrm{kHz}$. These frequencies are in the range of commercial audio telephone modems. The attenuation was not large enough to prevent commercial telephone modems from establishing communications (with an appropriate boost amplifier on the transmitter and/or receiver). This type of communication will require much more power (probably 100 times more power for the same distance).

From the communication protocol simulations, Optimized Link State Routing (OLSR) performed the best for a large scale network of pipeline sensors. While the data delivery rate was not quite as high as one would hope, especially at higher node speeds, delivery rate probably could be increased with a change of the OLSR parameters to allow more frequent neighbor sensing and multipoint relay set broadcast. With small networks, Dynamic Source Routing (DSR) would probably be a better choice because of its high data delivery rate.

While this project established the feasibility of both methods of signal transmission, the maximum limits are still unknown. The next step in this work is to test the feasibility of transmitting a few miles. For the waveguide transmission, the effect of a $5 \mathrm{GHz}$ transmission rate should also be assessed. 


\section{References}

Balanis, Constantine, Advanced Engineering Electromagnetics, John Wiley \& Sons, Inc., New York, NY, 1989.

Carson, J. R., "Wave Propagation in Overhead Wires with Ground Return, " Bell System J., vol. 5, pp. 539-555, Oct., 1926.

Erickson, K. T., A. Miller, E. K. Stanek, C. H. Wu, and S. Dunn-Norman. "Pipelines as Communication Network Links," Proceedings of Natural Gas Technologies II Conference, Phoenix, AZ, Feb. 8 - 11, 2004

Nestleroth, J.B., T. A. Bubenik and H. H. Haines, “The Pipeline Simulation Facilities for the Development of In-Line Inspection Technologies for Gas Transmission Pipelines," Materials Evaluation, Vol. 53, April 1995.

Sunde, Erling D., Earth Conductor Effects in Transmission Systems, Dover Publications, New York, NY, 1968.

\section{Acknowledgements}

Bruce Nestleroth, Stephanie Flamberg, and Bob Gertler of Battelle are acknowledged for their assistance in the tests performed at the Battelle pipeline simulation facility. This work was funded by the Department of Energy, National Energy Technology Laboratory, through contract DE-FC26-02NT41604. 


\section{Appendix A - PLC Programs}

For the transmission tests on the UMR pipeline, both programmable logic controllers were Allen-Bradley PLC-5/20E processors with an Ethernet connection. In this case, the PLC with the "WIRELESS22" program incremented its N7:0 location every second. The PLC with the program named "WIRELESS25" read the N7:0 integer from the PLC with the program named "WIRELESS22". Every time a change in N7:0 was detected by WIRELESS25, the O:2/00 discrete output channel was pulsed on for 0.5 seconds. Looking at the output module on the PLC, one observed the channel indicator for channel 0 flashing at about a $0.5 \mathrm{~Hz}$ rate when the communication between the two PLCs was without error.

WIRELESS22.RSP

LAD 2 - --- Total Rungs in File $=3$

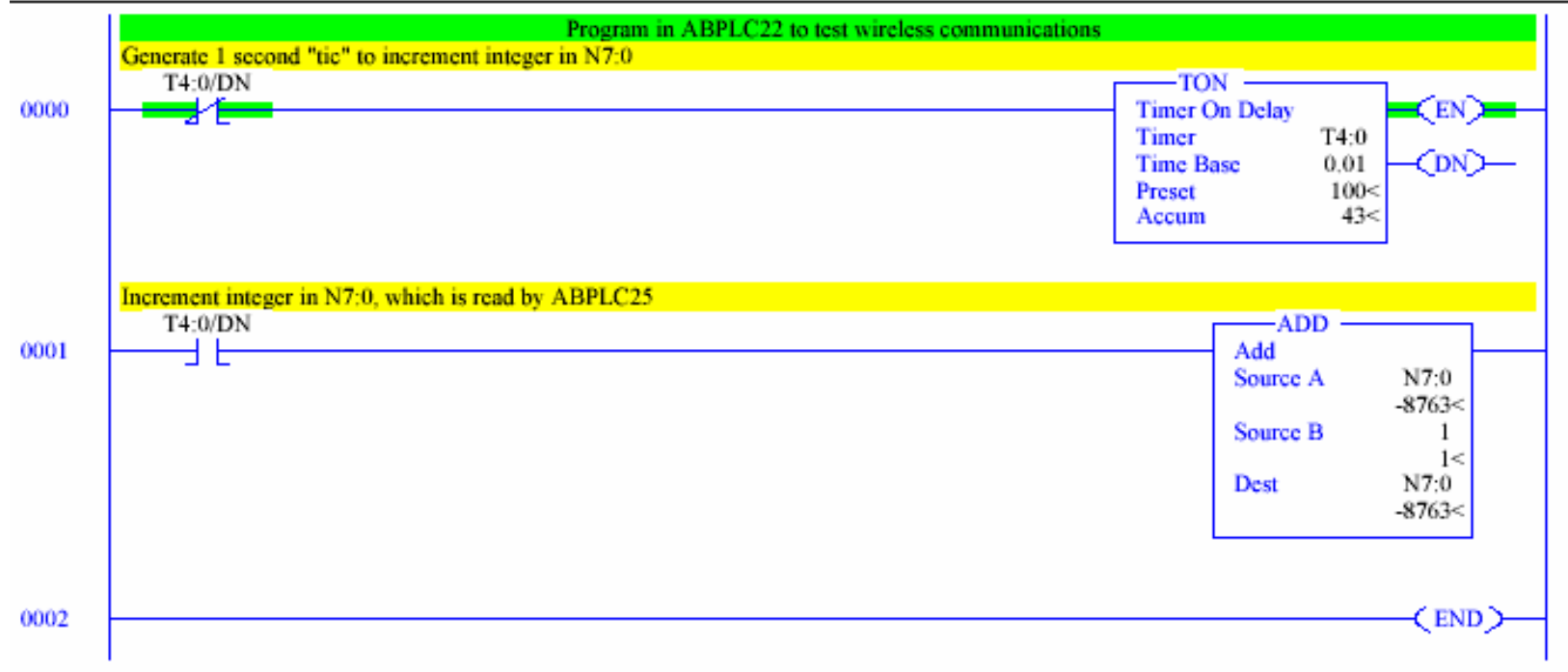


LAD 2 - -n Total Rungs in File $=6$

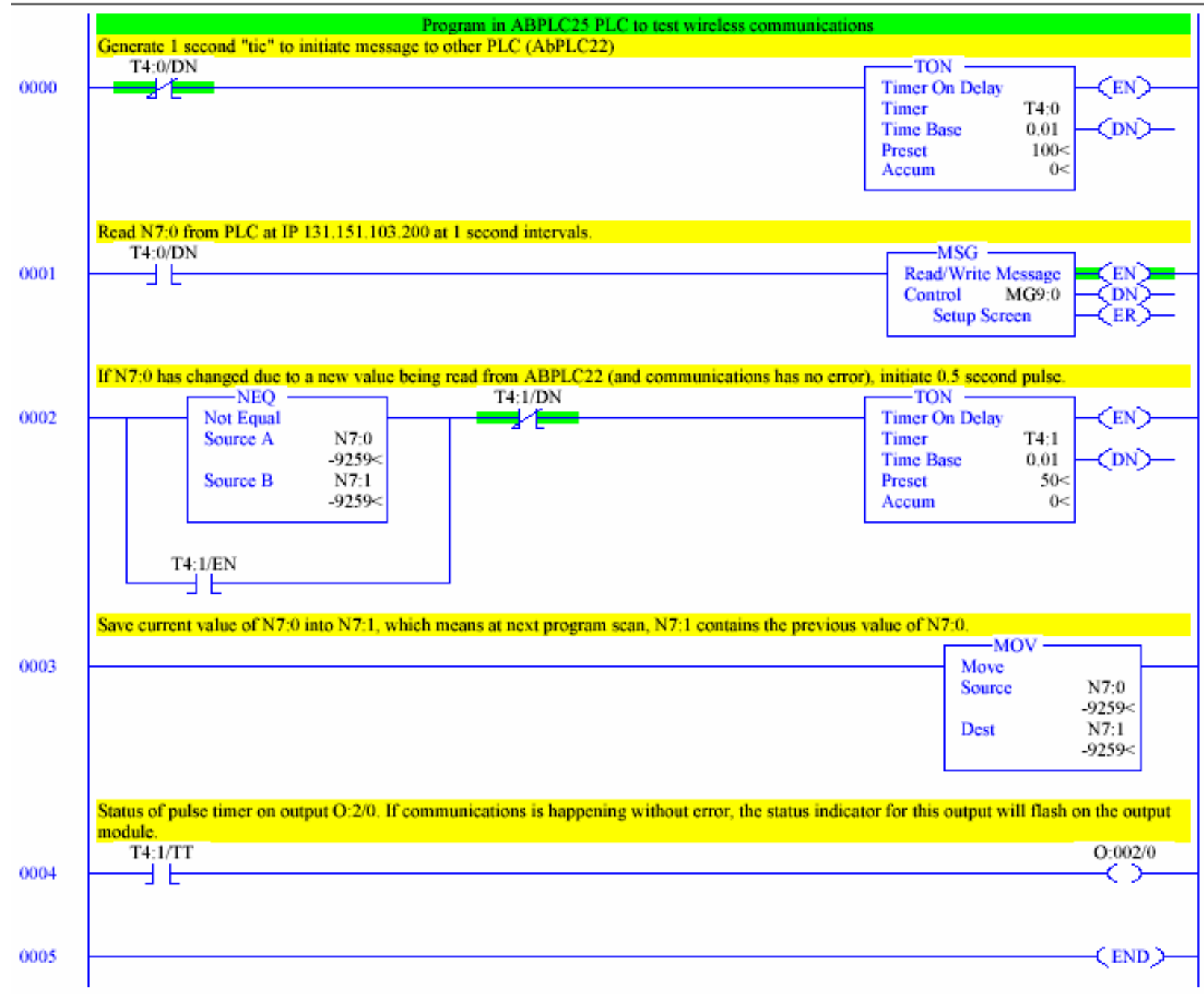

WIRELESS25.RSP

MSG Configuration

MSG - Fung \$2:1 - Ma9:0(2 Elements)

Communication Command: PLC-5 Typed Road

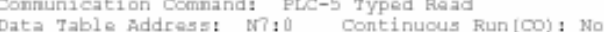

Iqnore if timed out (TO): No

Data Table Address:
Size in Elenents:

Data Table Address: $\mathrm{N}$ ?

Fthernat [IP] Address: 131.151 .103 .200 
For the transmission tests on the Battelle pipeline, both programmable logic controllers were Allen-Bradley SLC-5/05 processors with an Ethernet connection. In this case, the PLC with the "WIRELESS12" program reads the N7:1 integer from the PLC with the "WIRELESS13" program every second. The PLC with the program named "WIRELESS13" reads the N7:0 integer from the PLC with the program named "WIRELESS12" every second. The WIRELESS13 PLC copies bit 0 of N7:0 to bit 0 of N7:1. The WIRELESS12 PLC copies the inverse of bit 0 of N7:1 to bit 0 of N7:0. The net result is a "communications heartbeat." Bit 0 of N7:0 oscillates between 0 and 1 when there are no communication errors. For both PLCs, bit 0 of $\mathrm{N} 7: 0$ was copied to the O:2/00 discrete output channel. Looking at the output module on either PLC, one observed the channel indicator for channel 0 flashing at about a $0.5 \mathrm{~Hz}$ rate when the communication between the two PLCs was without error.

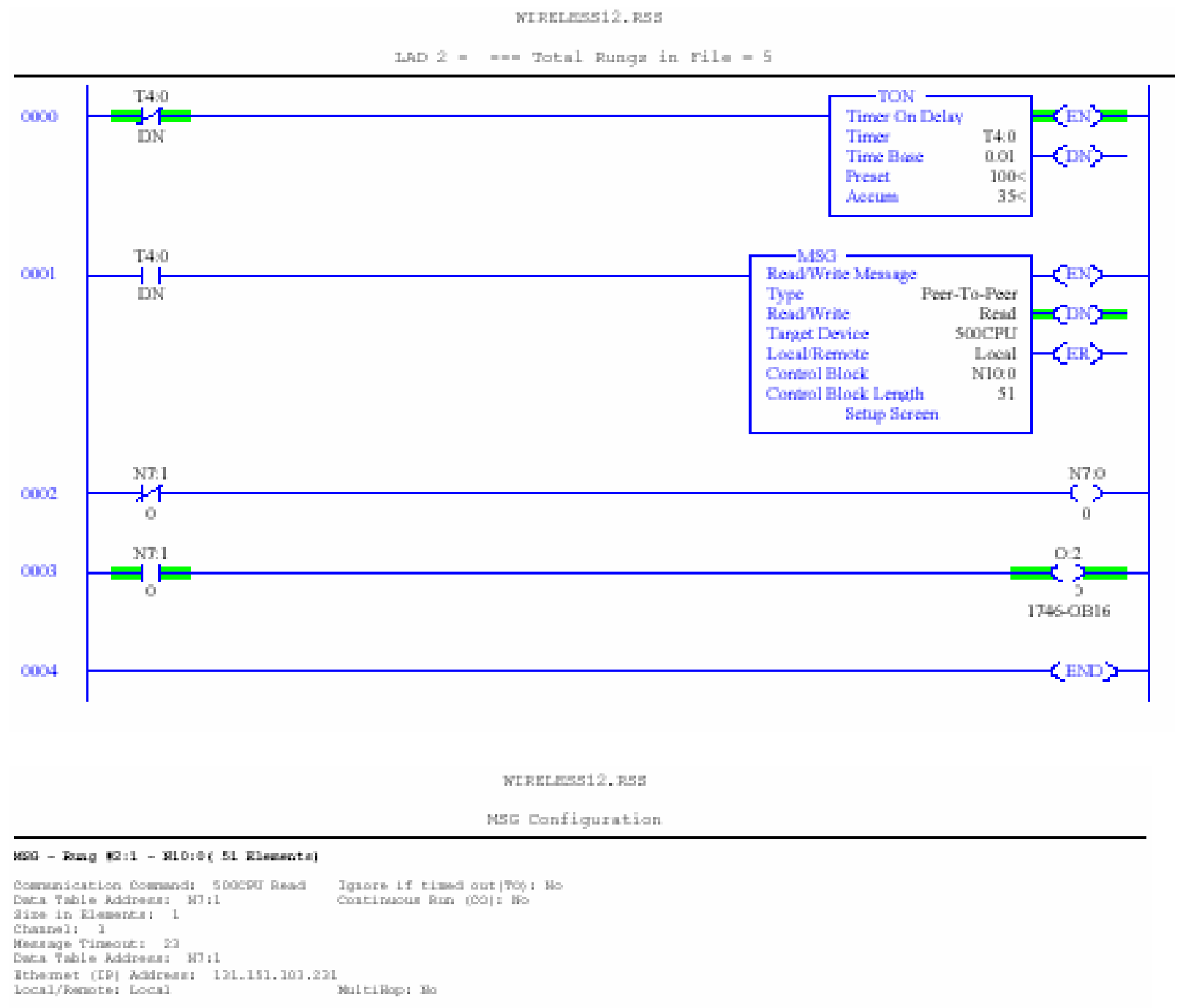


WIRELESS13.RSS

LAD 2 - --- Total Rungs in File = 5

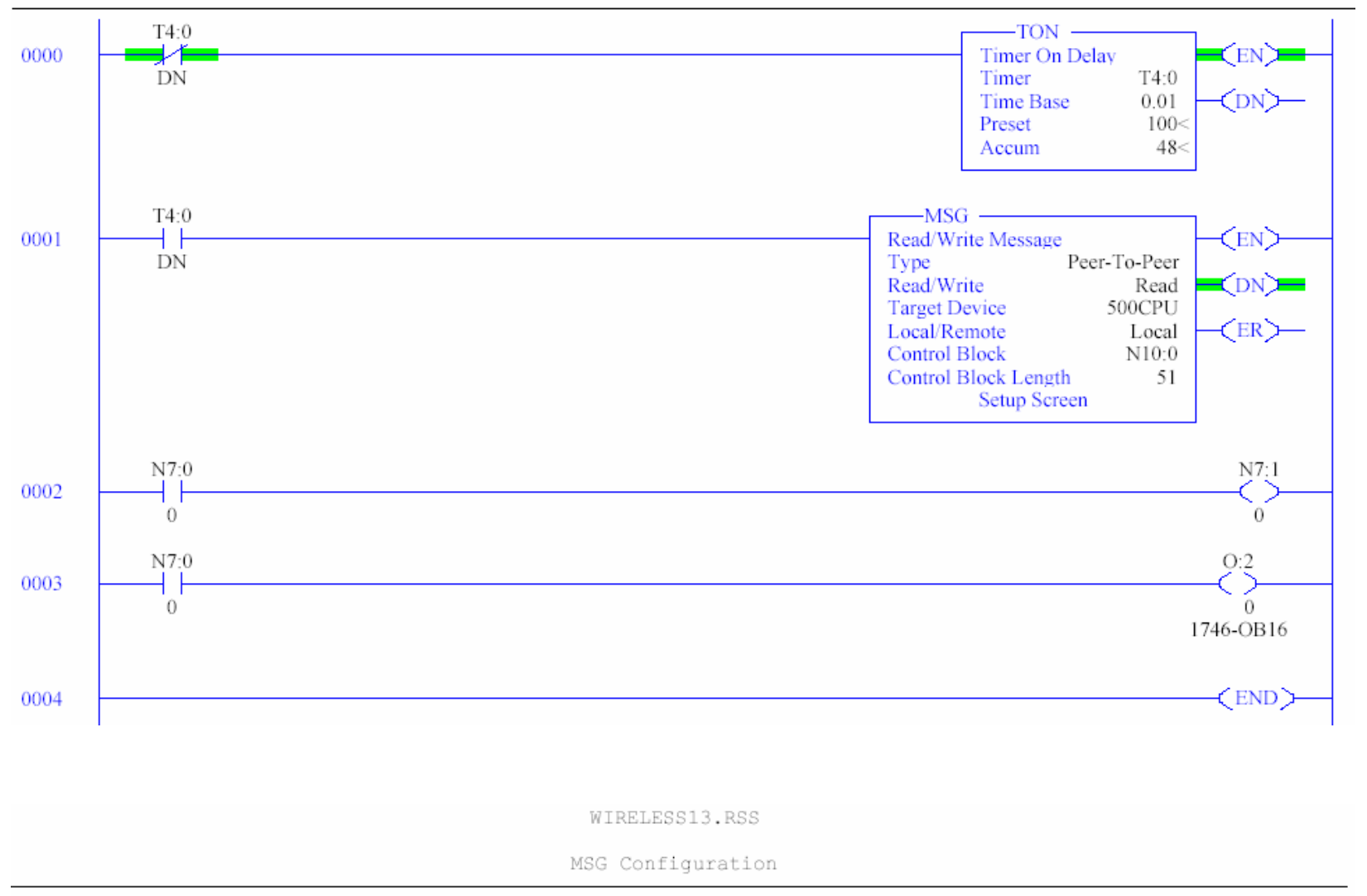

MSG - Rung \#2:1 - N10:0 ( 51 Elements)

Communication Command: 500CPU Read Ignore if timed out(TO) : No

Data Table Address: N7:0 Continuous Run (CO): No

Size in Elements: 1

Channel: 1

Message Timeout: 23

Data Table Address: N7:0

Ethernet (IP) Address: 131.151.103.218

Local/Remote: Local

MultiHop: No 


\section{Appendix B - Parametric Study Program}

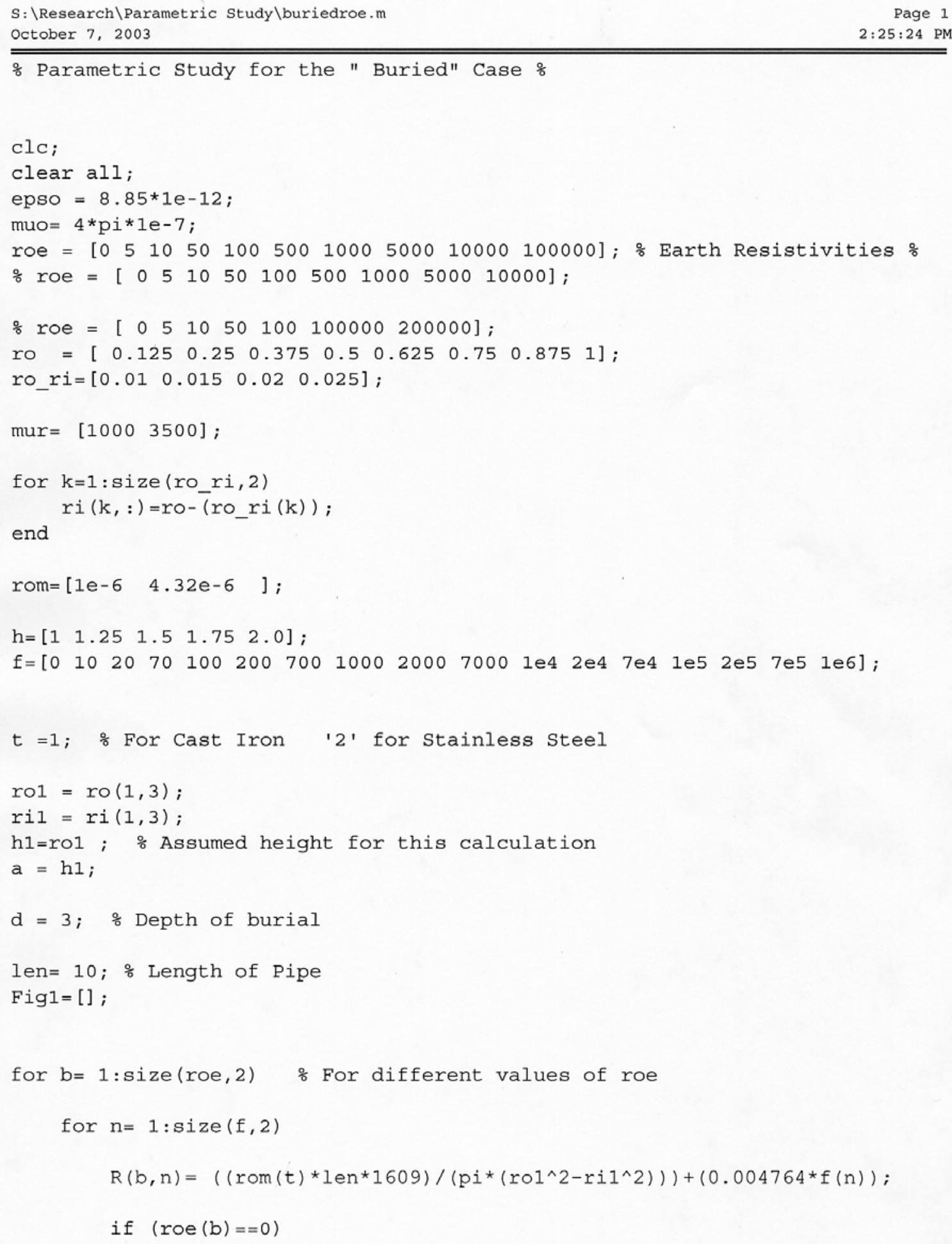




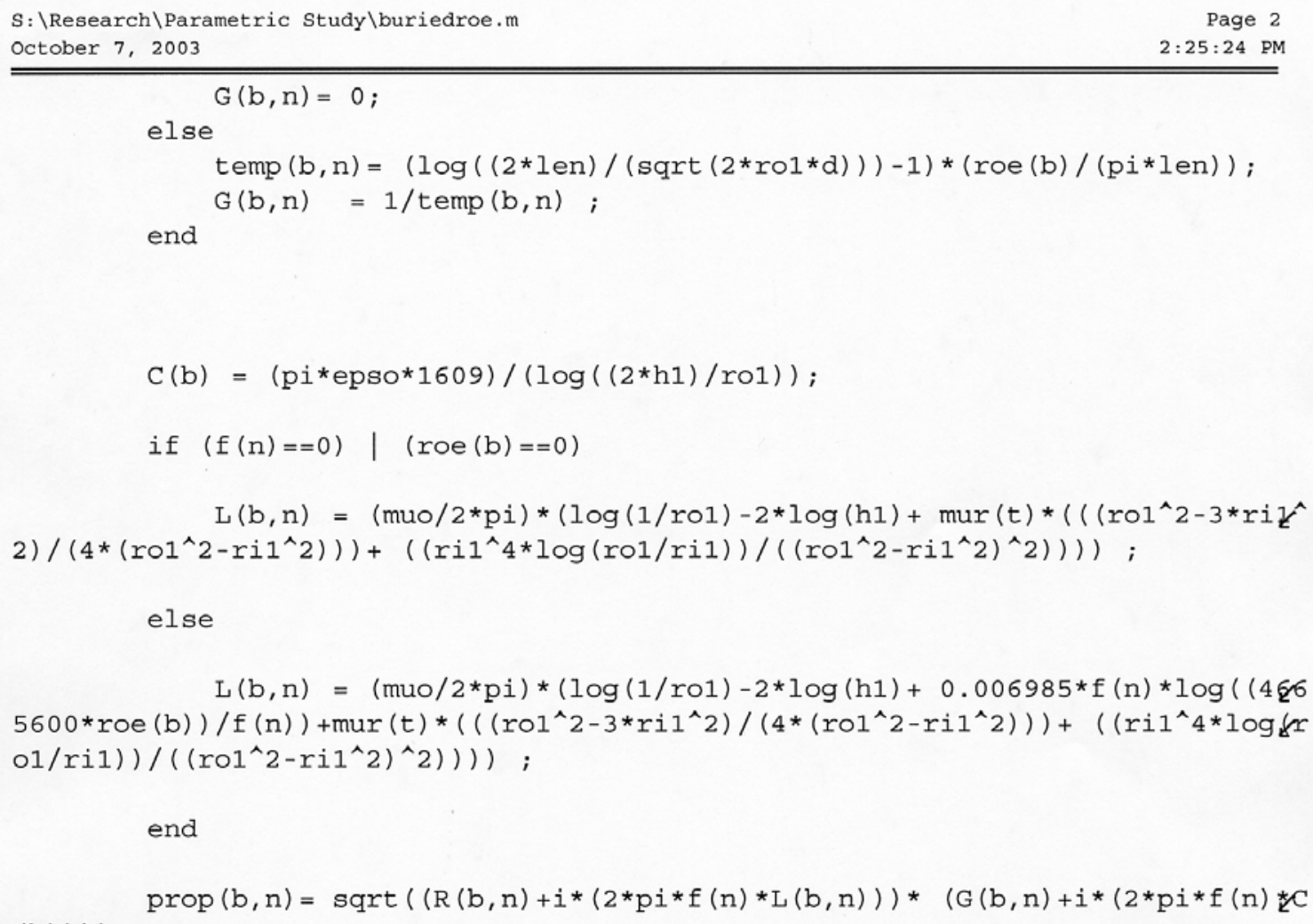


$S: \backslash$ Research $\backslash$ Parametric Study $\backslash$ buriedroe.m

Page 3 October 7, 2003

000 ', 'roe $=5000$ ', 'roe $=10000^{\prime}$ );

title('Effect of earth resistivity on Attenuation');

xlabel ('Frequency') ;

ylabel ('Attenuation') ; 